hep-th/0108100

RUNHETC-2001-24

\title{
D-Brane Instantons and K-Theory Charges
}

\author{
Juan Maldacena ${ }^{1,2}$, Gregory Moore ${ }^{3}$, Nathan Seiberg ${ }^{1}$ \\ ${ }^{1}$ School of Natural Sciences, \\ Institute for Advanced Study \\ Einstein Drive \\ Princeton, New Jersey, 08540 \\ 2 Department of Physics, Harvard University \\ Cambridge, MA 02138 \\ ${ }^{3}$ Department of Physics, Rutgers University \\ Piscataway, New Jersey, 08855-0849
}

\begin{abstract}
We discuss some physical issues related to the K-theoretic classification of D-brane charges, putting an emphasis on the role of D-brane instantons. The relation to Dinstantons provides a physical interpretation to the mathematical algorithm for computing K-theory known as the "Atiyah-Hirzebruch spectral sequence." Conjecturally, a formulation in terms of D-instantons leads to a computationally useful formulation of K-homology in general. As an application and illustration of this viewpoint we discuss some issues connected with D-brane charges associated with branes in WZW models. We discuss the case of $S U(3)$ in detail, and comment on the general picture of branes in $S U(N)$, based on a recent result of M. Hopkins.
\end{abstract}

July 27,2001 


\section{Introduction}

The present paper is intended to elucidate some physical aspects of the K-theoretic formulation of D-brane charge. In order to illustrate our general remarks we will apply these considerations to the question of classification of D-branes in WZW models. We summarize our results in the present introduction.

\subsection{Formulating K-theory using D-instantons}

D-brane charge is classified by K-theory [1, 2]. Many of the arguments for this are somewhat mathematical, and do not emphasize the physical difference between K-theoretic, as opposed to cohomological, classification of D-brane charges. One physical interpretation has been given by Witten in terms of brane-antibrane annihilation in [2] in the framework of the Sen conjectures [3]. The present note offers a slightly different (but related) viewpoint on the physical meaning of the K-theoretic classification of D-brane charges. We will focus on the following simple question: Suppose spacetime is a product $X=\mathbb{R} \times X_{9}$ where $X_{9}$ is a 9-dimensional space, possibly noncompact. What are the possible cycles $\mathcal{W} \subset X_{9}$ which can be wrapped by a D-brane? Broadly speaking, the answer to this question comes in two parts

(A.) The field theory on the D-brane must be consistent. For example, it must be anomaly free. This can put restrictions on the possible cycles on which "free D-branes" (i.e. D-branes with no other branes ending on them) can wrap.

(B.) We must identify branes which can be dynamically transformed into one another.

In the classification of D-brane charges in type II string theory the conditions (A) and (B) are implemented as follows:

(A) A D-brane can wrap $\mathcal{W} \subset X_{9}$ only if

$$
W_{3}(\mathcal{W})+\left.[H]\right|_{\mathcal{W}}=0 \quad \text { in } \quad H^{3}(\mathcal{W}, \mathbf{Z})
$$

Here $W_{3}(\mathcal{W})$ is the integral Stiefel-Whitney class 1 of $T \mathcal{W}$. In particular, in the DeRham theory $\left.[H]_{D R}\right|_{\mathcal{W}}=0$. A similar condition applies in other string theories. For

1 The reader will be able to follow the main points of this paper by ignoring this class (and ignoring Steenrod squares). We include such torsion classes for completeness. 
example, in the bosonic string we must impose condition (1.1) without the $W_{3}(\mathcal{W})$ term.

$(B)$ Branes wrapping homologically nontrivial $\mathcal{W}$ can nevertheless be unstable if, for some $\mathcal{W}^{\prime} \subset X_{9}$

$$
P D\left(\mathcal{W} \subset \mathcal{W}^{\prime}\right)=W_{3}\left(\mathcal{W}^{\prime}\right)+\left.[H]\right|_{\mathcal{W}^{\prime}}
$$

where the left hand side denotes the Poincare dual of $\mathcal{W}$ in $\mathcal{W}^{\prime}$. In other string theories, like the bosonic string, the question of stability is more complicated because they always include tachyons. It is not always simple to disentangle the instability associated with the ordinary tachyon, which is always present, from the instability of the brane.

(We remark parenthetically that in the cohomological classification of D-brane charges the principles $A, B$ are implemented as follows. First, free branes can wrap any homologically nontrivial cycle in $X_{9}$. Second, a brane wrapping a nontrivial cycle is absolutely stable. Thus, the homotopy classes of configurations of free branes can be labelled by $H_{c p t, *}\left(X_{9} ; \mathbf{Z}\right) \cong H^{*}\left(X_{9} ; Z\right)$. )

In section two of this paper we will justify conditions $(A)$ and $(B)$. Our point of view is not essentially new. Regarding (A), the role of global anomalies has already been thoroughly explained in [2,4,5]. Moreover, regarding (B), the phenomenon of brane instability was already noted in [6]. The novelty, such as it is, of the present note, is that we give a more precise description of the mechanism of K-theoretic brane instability. That mechanism is simply instability due to D-brane instanton effects, and the essential phenomenon goes back to Witten's formulation of the baryon vertex in the AdS/CFT correspondence [7].

In section three we go on to explain how conditions $(A)$ and $(B)$, are related to $\mathrm{K}$ theory. The essential point is that imposing conditions (1.1) "modulo" conditions (1.2) is closely related to a mathematical algorithm for the computation of $K$-theory known as the "Atiyah-Hirzebruch spectral sequence," (AHSS) including the AHSS in the presence of non-torsion $H$-fields. We therefore clarify the relation of the AHSS to physics.

To be more precise, the rules $(A)$ modulo $(B)$ are related to the computation of the AHSS at the third differential. It is important to stress, however, that the AHSS only gives an "approximation" to K-theory since it is predicated on a filtration and one must then solve an extension problem. Moreover, while the rules "(1.1) modulo (1.2)" are related to the cohomology of the third AHSS differential, they are on the one hand stronger, and on 
the other hand, do not involve the use of higher differentials. Conjecturally, a systematic implementation of the rules $(A)$ modulo $(B)$, including the possibility of branes within branes, is a complete formulation of K-homology. The results of section 4 provide some evidence for this conjecture.

Our improved understanding of the physical basis for the AHSS clarifies the physical basis for using the mathematical group $K_{H}^{*}(X)$ for the classification of D-brane charges in the presence of a cohomologically nontrivial $H$-field. While it is perhaps well-accepted that $K_{H}^{*}(X)$ classifies D-brane charges when $H$ is nontorsion it is worth understanding the physical basis of this claim more thoroughly. In his original proposal [2] Witten gave an argument which only applied to the case when $[H]$ is a torsion class. A more direct argument was given by Kapustin [8], developing the ideas of [4]. Unfortunately, Kapustin's method again only applies for $[H]$ torsion. It would be extremely interesting to formulate a direct generalization of his argument to the nontorsion case but this appears to be nontrivial. In [9] P. Bouwknegt and V. Mathai pointed out that the mathematical theory of $K_{H}^{*}(X)$ makes perfectly good sense when $H$ is nontorsion and moreover has a natural formulation in terms of $C^{*}$ algebras. (For further useful discussion along these lines we recommend the paper of Mathai and Singer [10]. See also [11].) While these discussions fit in well with our current understanding of D-branes, as discussed in [12, 13], they do not demonstrate the physical relevance of $K_{H}^{*}(X)$.

By giving a clearer physical foundation for the AHSS for nontorsion $H$-fields we are giving further support to the general claim that $K_{H}^{*}(X)$ is the correct D-brane charge group. In fact, our clarification of the physical basis of $K_{H}^{*}(X)$ leads to some insights into the limitations of this group as a group of brane charges. In a companion paper [14] we will examine critically the physical meaning of $K_{H}^{*}(X)$ in the larger context of M-theory.

Finally, we would like to clarify our use of the term "instanton." The D-branes fall into superselection sectors which are labeled by their charges. The configurations which we refer to as instantons represent transitions between different such sectors, and therefore they identify them. Consider a transition from a brane configuration $A_{i}$ to a brane configuration $A_{f}$. It is achieved by an interpolation $A(t)$ with the boundary conditions $A\left(t_{i}\right)=A_{i}$ and $A\left(t_{f}\right)=A_{f}$. We refer to the interpolation $A(t)$ as an instanton. It is also a D-brane in space $X_{9}$.

Our discussion depends only on the topology of $X_{9}$ and of the various branes $A$ and $A(t)$ and not on their detailed geometry. If more information about the dynamics is given, then the D-branes $A$ should be stationary solution of the equations of motion, and $A(t)$ 
must satisfy the time dependent equations of motion where the parameter $t$ is interpreted as the time. Then an important distinction should be made between two situations regarding the interpolation $A(t)$ :

1. When the transition between $A_{i}$ and $A_{f}$ is a classically allowed transition the interpolation $A(t)$ should satisfy the equations of motion with Lorentzian signature time.

2. When the transition between $A_{i}$ and $A_{f}$ involves tunneling and is not allowed classically $A(t)$ should satisfy the equations of motion with Euclidean signature time $t$.

The traditional use of the term "instanton" is only for tunneling transitions but we will use the term for every interpolation.

\subsection{Applications to D-branes in WZW models}

WZW models are the preeminant example of string backgrounds with cohomologically nontrivial nontorsion $H$-flux. Moreover, they are solvable conformal field theories. They are therefore very natural examples in which to study ideas of the connection between D-branes and K-theory. In section 4 we use the description of allowed brane wrappings ("rule $A$ modulo rule $B$ ") to compute $K_{H}^{*}(S U(2))$ and $K_{H}^{*}(S U(3))$. While $K_{H}^{*}(S U(2))$ is well-known, the result for $S U(3)$ is new. We discuss in detail some of the necessary topology of $S U(3)$ in order to establish the result.

We then proceed to interpret the $S U(3)$ classes in terms of D-branes. In order to do this we review (and slightly extend) the beautiful theory of D-branes in group manifolds which has been developed by several authors over the past few years. This we do in sections five and six.

In section 7 we introduce a new set of D-branes in the WZW theory which we call "parafermionic branes." The discussion here is a programmatic description of an extension of the ideas of [15]. Much work remains to be done.

In section 8 we compare the CFT results on D-branes with those of K-theory. In particular, we describe a recent result of M. Hopkins computing $K_{H}^{*}(S U(N))$ for all $N$. The result of Hopkins fits in very naturally and beautifully with the physical picture of D-branes in WZW theory, although the detailed CFT construction of a set of D-branes producing the full set of K-theory charges remains to be done. 


\section{Physical justification of conditions $(A)$ and $(B)$}

The condition $(A)$ is well-known. In the DeRham theory it simply comes about since the $H$-field must be trivialized on the D-brane by the equations of motion $\left.H\right|_{\mathcal{W}}=d(F+B)$, and hence $\left.[H]\right|_{\mathcal{W}}=0$. At the level of integral cohomology the condition (1.1) follows from the cancellation of global anomalies for fundamental open strings ending on the D-brane, as shown in [4, [8].

To explain $(B)$, suppose there is a cycle $\mathcal{W}^{\prime} \subset X_{9}$ on which

$$
W_{3}\left(\mathcal{W}^{\prime}\right)+\left.[H]\right|_{\mathcal{W}^{\prime}} \neq 0 .
$$

As we have just explained, we cannot wrap a D-brane on $\mathcal{W}^{\prime}$. Let us do so anyway, with a D-brane instanton .

We can then cancel the global anomalies on the D-brane worldvolume $\mathcal{W}^{\prime}$ by adding a magnetic source for $F$ on $\mathcal{W} \subset \mathcal{W}^{\prime}$ such that

$$
P D\left(\mathcal{W} \subset \mathcal{W}^{\prime}\right)=W_{3}\left(\mathcal{W}^{\prime}\right)+\left.[H]\right|_{\mathcal{W}^{\prime}}
$$

The proof will be given presently. A D-brane ending on $\mathcal{W}$ provides just such a magnetic source. Therefore, we arrive at the following picture: A brane wrapping a spatial cycle $\mathcal{W}$ propagates in time, and terminates on a D-instanton wrapping $\mathcal{W}^{\prime}$. This means the brane wrapping the spatial cycle $\mathcal{W}$ can be unstable, and decays due to D-brane instantons wrapping $\mathcal{W}^{\prime}$. This is illustrated in figure 1 .

One should note that condition $(B)$ only means the brane wrapping $\mathcal{W}$ might decay. The brane wrapping $\mathcal{W}$ could be carrying other conserved charges that prevent decay to the vacuum. This is related to the fact that we are only answering the question of which cycles can be wrapped, and not addressing the full question of which branes within branes are allowed. The latter question is indeed answered by K-theory, and could be analyzed by systematic use of the above viewpoint. 


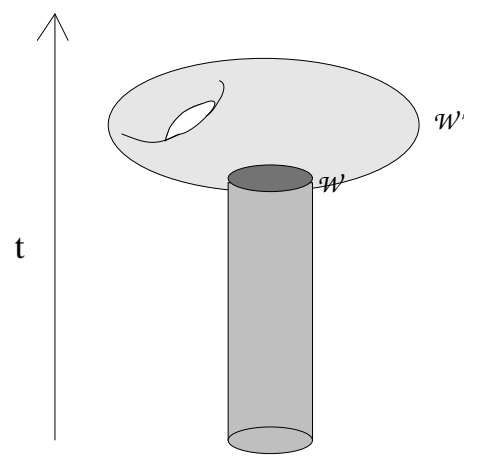

Fig. 1: A D-brane instanton wraps $\mathcal{W}^{\prime}$ which does not satisfy (1.1). Another D-brane world volume terminating on a cycle $\mathcal{W} \subset \mathcal{W}^{\prime}$ satisfying (1.2) is unstable (provided it carries no other D-brane charges), and decays to the vacuum.

Let us now indicate why (2.2) really does cancel the anomalies. (See [5] for a more complete discussion.) In the DeRham theory this is easy, since by definition of a magnetic source $d F=2 \pi P D\left(\mathcal{W} \subset \mathcal{W}^{\prime}\right)$. To extend the argument to the integral condition we proceed as follow 22. For simplicity we put $H=0$, but allow $W_{3}\left(\mathcal{W}^{\prime}\right)$ to be nonzero.

Suppose a D-brane instanton wraps $\mathcal{W}^{\prime}$ with $W_{3}\left(\mathcal{W}^{\prime}\right)$ nonzero. Suppose another Dbrane wraps $\mathcal{W} \times R_{+}$and terminates on $\mathcal{W}^{\prime}$.

We claim that in this situation we cancel the Freed-Witten anomaly if $P D(\mathcal{W} \rightarrow$ $\left.\mathcal{W}^{\prime}\right)=W_{3}\left(\mathcal{W}^{\prime}\right)$. Recall that the Freed-Witten anomaly is based on the sign ambiguity in the definition of the of the path integral for worldsheet fermions. We thus consider a family of open string worldsheets $S_{t}, 0 \leq t \leq 1$, ending on $\mathcal{W}^{\prime}$, where $S_{0}=S_{1}$. The boundaries of these worldsheets $\partial S_{t}$ sweep out a 2-cycle $\Sigma_{2}=\partial S \subset \mathcal{W}^{\prime}$, where $S$ denotes the total space of the family. As shown in [4], the holonomy of the fermion determinants around $S^{1}$ is a sign

$$
\exp \left[i \pi\left\langle w_{2}\left(\mathcal{W}^{\prime}\right),\left[\Sigma_{2}\right]\right\rangle\right]
$$

Freed and Witten then extend the condition to require that this factor be unity for any 2-cycle $\Sigma_{2} \subset \mathcal{W}^{\prime}$. To proceed we will assume, again for simplicity, that the anomaly is due to a 2 -cycle $\Sigma_{2}$ with $2 \Sigma_{2}=\partial D_{3}$ for some chain $D_{3} \subset \mathcal{W}^{\prime}$. We can learn about $\Sigma_{2}$ by reviewing the definition of the mod-two Bockstein map relating $w_{2}$ and $W_{3}$. Recall that we begin by lifting $w_{2}$ to an integral cochain $\bar{w}_{2}$ such that

$$
\left\langle w_{2}, C_{2}\right\rangle=\left\langle\bar{w}_{2}, C_{2}\right\rangle \bmod 2
$$

2 The reader uninterested in torsion should skip the remainder of this section. 
for any 2-chain $C_{2}$. Now $\left\langle\bar{w}_{2}, \Sigma_{2}\right\rangle$ is an integer. If $2 C_{2}=\partial D_{3}$ we can now say:

$$
2\left\langle\bar{w}_{2}, C_{2}\right\rangle=\left\langle\delta \bar{w}_{2}, D_{3}\right\rangle=2\left\langle W_{3}, D_{3}\right\rangle
$$

by definition of the Bockstein, and, this being an equation of integers, we can divide by 2 to get

$$
\left\langle\bar{w}_{2}, C_{2}\right\rangle=\left\langle W_{3}, D_{3}\right\rangle \bmod 2
$$

Now let us apply this to our cycle $\Sigma_{2}$. Note that $\left\langle W_{3}, D_{3}\right\rangle$ is the oriented intersection number of $D_{3}$ with $P D\left(W_{3}\right)$. So we see that an anomaly will appear when there is a cycle $\Sigma_{2}$ that links the worldvolume $\mathcal{W}$ of the terminating D-brane. In the presence of a magnetic source there is an extra term in the path integral, morally of the form $\exp \left[i \int_{\partial S_{t}} A\right]$, where $S_{t}$ is the open string worldsheet. This extra factor cancels the ambiguity in the fermion determinant since

$$
\int_{\partial D_{3}} F / 2 \pi=1 \Rightarrow \int_{\Sigma_{2}} F / 2 \pi=1 / 2
$$

and hence the magentic source along $\mathcal{W}$ leads to a factor $\exp \left[i \int_{\Sigma_{2}} F\right]=-1$, cancelling the ambiguous sign in the fermion determinant.

\subsection{Examples}

In this section we illustrate the above discussion with a few examples.

Consider first the case of D0 brane number in a spacetime with no torsion: The worldvolume of $N D 0$ 's defines a 0 -cycle $W_{0}$ which is Poincare dual to $N x_{9}$ where $x_{9}$ generates $H_{9}(X ; Z)=Z$. 目 Naturally enough, we identify $N$ with the 0 -brane number in the approximation $H_{c p t}^{\text {odd }} \cong K^{1}$. However if $\Sigma_{3}$ is any 3-cycle in spacetime on which $\int_{\Sigma_{3}} H=$ $k$, say, then we can consider a $D 2$ instanton wrapping $\Sigma_{3}$. This instanton will violate D0 charge since $k$ worldlines of $D 0$ branes must end on $\Sigma_{3}$. The reason is that these end on monopoles ( $=2+1$ dimensional instantons) for the $U(1)$ fieldstrength of the $D 2$ brane so if $k$ worldlines of $D 0$ 's end on a $D 2$ at positions $x_{i} \in \Sigma_{3}$ then $d F=2 \pi \sum_{i=1}^{k} \delta^{3}\left(x-x_{i}\right)$. In terms of cohomology we are identifying

$$
N x_{9} \sim N x_{9}+H \wedge P D\left(\Sigma_{3}\right)
$$

and since $\int_{\Sigma_{3}} H=k$ we know that

$$
H \wedge P D\left(\Sigma_{3}\right)=k x_{9}
$$

3 We assume $X$ is connected. It is necessarily oriented. 
so that we have an identification $N \sim N+k$. Consequently, $D 0$ brane number is only defined modulo $k$, and the charge group has (at least) $k$-torsion.

The same phenomenon shows that other D-instantons induce $k$-torsion for other charges. For example, a $D 2$ brane wrapping $\sum_{i} n_{i} w_{i}$, where $w_{i}$ form a basis of $H_{2}(X, Z)$,

has a Poincare dual 7 -form $\omega_{7}=n_{i} \omega_{7}^{i}$. Now we should worry about $D 4$ instantons wrapping various five cycles. For example, D4 instantons wrapping $\Sigma_{5}=\left(\sum_{i} a_{i} w_{i}\right) \times \Sigma_{3}$ lead to the identification $n_{i} \sim n_{i}+k a_{i}$. The reason is the same as before: A D4 instanton wrapping the 5 -manifold $\Sigma_{5}$ violates the rule $[H]=0$. To account for the trivialization $d \mathcal{F}=d F+H$ we must have $D 2$-brane worldvolumes ending on the homology class $k\left(\sum a_{i} w_{i}\right)$ in $\Sigma_{5}$.

As an example with torsion in $H_{c p t}^{*}(X, \mathbf{Z})$, and to make contact with [6] let us consider a simple example with $H=0$. Suppose that $c=S q^{3}\left(c_{0}\right)$ for some $c_{0} \in H^{3}(X, Z)$. Then $c$ is $S q^{3}$ closed, and hence corresponds to some K-theory class. Nevertheless, it is also $S q^{3}$ exact, and hence its K-theory lift is zero. Since its K-theory charge vanishes we expect a brane with $P D(c)=\mathcal{W}$ to decay. Physically, $P D(c)=Q_{3}$ is the homology class of a spatial section of a D3-brane, while $P D\left(c_{0}\right)=Q_{6}$ is the homology class of a cycle which will be wrapped by a D5-brane instanton. Since $S q^{3}\left(c_{0}\right)$ is nonzero, $Q_{6}$ is not $S p i n^{c}$. As we have explained, we can cancel the global anomalies by allowing the worldvolume $Q_{3}$ of the D3 brane to end on $Q_{6}$, provided $P D\left(Q_{3} \hookrightarrow Q_{6}\right)=W_{3}\left(Q_{6}\right)$.

\section{Relation of conditions $(A)$ and $(B)$ to K-theory}

In this section we will argue that conditions $(A)$ and $(B)$ are in fact conditions of $K$-theory. We will show this by demonstrating that $(A)$ and $(B)$ are refinements of the procedure of taking $d_{3}$-cohomology in the AHSS for $K_{H}^{*}(X)$.

\subsection{Review: The mathematical Formulation of the AHSS}

Let us briefly review the AHSS. (For further background see [6].)

A $K$-theory class $x$ in $K^{*}(X)$ determines a system of integral cohomology classes. If $x \in K^{0}(X)$ these are the Chern classes $c_{i}(x) \in H^{2 i}(X, \mathbf{Z})$. If $x \in K^{1}(X)$ there are classes $\omega_{2 i+1} \in H^{2 i+1}(X, \mathbf{Z})$ related to Chern-Simons invariants.

Conversely, given such a system of cohomology classes we may ask whether it came from a K-theory class. The answer, in general, is "no." The AHSS is a successive approximation scheme for computing the necessary relations on the classes. This amounts to the following algorithm: 
a.) In the first approximation

$$
\begin{aligned}
K^{0}(X) \sim E_{1}^{\text {even }}(X) & :=\oplus_{j \text { even }} H^{j}(X, \mathbf{Z}) \\
K^{1}(X) & \sim E_{1}^{\text {odd }}(X):=\oplus_{j \text { odd }} H^{j}(X, \mathbf{Z})
\end{aligned}
$$

b.) Then, for a certain differential 1 , $d_{3}: H^{j}(X, Z) \rightarrow H^{j+3}(X ; Z)$, we compute

$$
E_{3}^{j}(X):=\left.\operatorname{ker} d_{3}\right|_{H^{j}} /\left.\operatorname{Im} d_{3}\right|_{H^{j-3}}
$$

and set $E_{3}^{\text {even }}=\oplus_{j}$ even $E_{3}^{j}(X)$, etc. to obtain the first correction:

$$
\begin{aligned}
K^{0}(X) \sim E_{3}^{\text {even }}(X) & :=\left(\text { Ker }\left.d_{3}\right|_{H^{\text {even }}}\right) /\left(\left.\operatorname{Im} d_{3}\right|_{H^{\text {odd }}}\right) \\
K^{1}(X) \sim E_{3}^{\text {odd }}(X) & :=\left(\left.\operatorname{Ker} d_{3}\right|_{H^{\text {odd }}}\right) /\left(\left.\operatorname{Im} d_{3}\right|_{H^{\text {even }}}\right)
\end{aligned}
$$

c.) Then, for a certain differential, $d_{5}: E_{3}^{j}(X, Z) \rightarrow E_{3}^{j+5}(X ; Z)$, we compute

$$
E_{5}^{j}(X):=\left.\operatorname{ker} d_{5}\right|_{E_{3}^{j}} /\left.\operatorname{Im} d_{5}\right|_{E_{3}^{j-5}}
$$

to obtain the next approximation, and so on.

d.) One keeps computing cohomology in this way to get $E_{\infty}^{j}(X)$. (The procedure is guaranteed to stop after a finite number of steps if $X$ is finite dimensional). The main theorem states that the "associated graded group" $G r(K)$ is given by

$$
\begin{aligned}
& G r\left(K_{H}^{0}(X)\right)=\oplus_{j} E_{\infty}^{2 j}(X) \\
& G r\left(K_{H}^{1}(X)\right)=\oplus_{j} E_{\infty}^{2 j+1}(X)
\end{aligned}
$$

We will explain the notation $G r(K)$ presently. In "good cases" we can identify $G r(K)=K$. See remarks below for the meaning of "good." The AHSS is useful because it is a clearly defined computational algorithm, but it does have some drawbacks.

The first drawback is that one plainly needs to have a useful expression for the differentials $d_{3}, d_{5}, \ldots$. 目 $\mathrm{A}$ simple expression for the differential $d_{3}$ is known. In ordinary K-theory it was identified in [17] as $d_{3}=S q^{3}$. In [18] it was identified for twisted $K$-theory

\footnotetext{
4 The word "differential" means $\left(d_{3}\right)^{2}=0$

5 The initial term of the spectral sequence is $C^{*}\left(X, h^{*}(p t)\right)$, for any generalized cohomology theory $h^{*}$. Thus, following the general procedure in [16] chapter XV, one could in principle extract the higher differentials. This appears to be extremely difficult in practice.
} 
as $d_{3}=S q^{3}+H$, in the context of $C^{*}$-algebra theory. This formula was rediscovered in [6,19]. Note that in the DeRham theory we obtain the simple expression

$$
d_{3}(\omega)=[H] \wedge \omega
$$

Not much is known about the higher differentials in general. There are scattered results for $H=0$, and it appears that nothing further is known for $H$ nonzero. Fortunately, on compact spin 10-folds at $H=0$ the higher differentials are not needed [6].

A second drawback is that in general the AHSS only gives an "approximation" to $K_{H}^{*}(X)$, in the following sense. Suppose we have a cell-decomposition, or simplicial decomposition $X_{0} \subset X_{1} \subset \cdots \subset X_{n}$. Then we define $K_{(p)}^{*}(X)$ to be the classes which become trivial upon restriction to $X_{p}$. Obviously $K_{(p+1)}^{*}(X) \subset K_{(p)}^{*}(X)$. The AHSS really computes the "associated graded space" which is, by definition

$$
\begin{aligned}
& G r\left(K_{H}^{0}(X)\right)=\oplus_{p} K_{H,(p)}^{0}(X) / K_{H,(p+1)}^{0}(X) \\
& G r\left(K_{H}^{1}(X)\right)=\oplus_{p} K_{H,(p)}^{1}(X) / K_{H,(p+1)}^{1}(X)
\end{aligned}
$$

In passing from (3.7) to the full $K$-theory group one needs to solve an extension problem to obtain the correct torsion subgroup. Fortunately, in many cases of interest the extension problem is either not too severe or even absent. However, there are important examples, such as compact Lie groups of rank greater than two, and homogeneous spaces, where this complication can be significant. In the physical context, the extension problem can be quite important in the presence of orientifolds. See [20] for a very interesting discussion of this point.

\subsection{Physical interpretation of the AHSS}

In this section we will explain the relation between taking $d_{3}$ cohomology in the AHSS and imposing the conditions $(A)$ modulo $(B)$.

Recall we are working on spacetimes of the form $\mathbb{R} \times X_{9}$. Consider a brane wrapping a $p$-manifold $\mathcal{W} \subset X_{9}$. One may associate to $\mathcal{W}$ several topological classes. First and most obviously, $\mathcal{W}$ has an associated homology cycle $Q(\mathcal{W}) \in H_{p}\left(X_{9}, Z\right)$. Since $X_{9}$ is oriented there is a Poincare dual integral cohomology class $\eta(\mathcal{W}) \in H_{c p t}^{9-p}\left(X_{9}, Z\right)$. Moreover, the brane wrapping $\mathcal{W}$ has gauge fields and consequently the D-brane charge is really associated with a class in the $K$-theory of $X_{9}$. The homology class $Q$ can be extracted from the $K$ theory class, since $Q$ represents the "support" of the K-theory class. In physical terms, 
if the D-brane is realized by tachyon condensation [3], the support is the locus where the tachyon field vanishes. We can then obtain a cohomology class from $\eta=P D(Q)$. More generally, to a collection $\left(\omega_{1}, \omega_{3}, \ldots, \omega_{9}\right)$ we associated a collection of branes by taking Poincaré duals to obtain a collection of spatial cycles $\left(P D\left(\omega_{1}\right), \ldots, P D\left(\omega_{9}\right)\right)$ around which $D 8, D 6, \ldots, D 0$-branes are wrapped.

The interpretation of the kernel of $d_{3}$ is known from [6]: A necessary condition for a cohomology class $\omega \in H_{c p t}^{j}\left(X_{9} ; Z\right)$ to survive to $E_{\infty}^{j}$ is that $d_{3}(\omega)=\left(S q^{3}+[H]\right) \omega=0$. Clearly this is implied by the anomaly cancellation condition (1.1). We therefore interpret $d_{3}(\omega)=0$ as a (partial) requirement of global anomaly cancellation. In fact, it is weaker than $(1.1)$.

A natural question 6 now arises: How should we interpret the quotient in (3.2) by the image of $d_{3}$ ? That is, what is the physics of the identification $\omega \sim \omega+\left(S q^{3}+H \cup\right) \omega^{\prime}$ in $E_{3}^{j}$ where $\omega^{\prime}$ is a class of degree $(j-3)$ ? From the discussion in section 2 it is clear that the physical interpretation is that this accounts for charge violation due to $D$-instanton effects. Again, dividing by the image of $d_{3}$ is weaker than the full condition (1.2).

Remark We conclude with one remark. In the AHSS discussion we need to choose a filtration of $K(X)$ (from a cell decomposition) and we also need the higher differentials. On the other hand, in the physical discussion we didn't make any use of filtrations, nor of higher differentials. It would be nice to clarify the relation between these two procedures. Indeed, one could probably deduce what the differential $d_{5}$ must be by careful examination of conditions $(A)$ and $(B)$. In this connection it is worth noting that $K$-theory is not easily described by the cohomology of a natural functor from the category of topological spaces to chain complexes.

\section{Application to WZW models: Computing $K_{H}^{*}(S U(2))$ and $K_{H}^{*}(S U(3))$}

Conformal field theories based on WZW models can be used to construct vacua of type II string theory. The simplest examples involve a free time coordinate, a level $k$ WZW model of $G=S U(2), S U(3)$, or $S O(4)$, and other fields. The restriction to $S U(2), S U(3)$ or $S O(4)$ comes from the requirement that the total contribution to $\hat{c}$ from the spatial part of the conformal field theory must be at most nine. Other groups can be used in a similar way provided their rank is less than nine and their level $k$ is not too large. Actually, a

6 This question first came up in a discussion with R. Dijkgraaf, July 1999. 
slight extension of this framework allows us to use all possible groups with all values of $k$. The point is that if the dilaton varies linearly in time, its contribution to $\hat{c}$ is less than one. Allowing such behavior of the dilaton, there is no restriction on $\hat{c}$ of the spatial part of the theory and all groups can be used.

Let us examine the D-branes in $S U(2)$ and $S U(3)$ in more detail.

\section{1. $S U(2)$}

In the level $k S U(2)$ WZW model we have $[H]=k x_{3}$ where $x_{3}$ is an integral generator of $H^{3}(S U(2) ; Z)=Z$. The computation of $K_{H}^{*}(S U(2))$ from the AHSS is completely straightforward and yields $Z / k Z$. (See [10] for the computation in the $C^{*}$ algebra context.) This is easily understood in terms of the general discussion of section 2: The D0 brane charge is violated by the D2 instantons.

This result is, of course, very closely related to results discussed in the extensive literature on branes in WZW models 21-31. We will comment on some relations to the literature in section 5 below.

The group $S U(2)$ is not sufficiently complicated topologically to illustrate some important issues in the classification of D-branes in WZW models. Some very interesting novelties already arise when we try to extend the discussion to the case of the level $k$ $S U(3)$ WZW model. In the next two sections we will discuss the relation of D-branes to K-theory for the $S U(3)$ model.

\subsection{Branes in $S U(3)$ : Some topological preliminaries}

In this section we summarize some topological facts which will be useful in understanding the charge groups for D-branes in $S U(3)$ WZW models.

It is well-known that $S U(n)$ is a twisted product of odd spheres. Rationally, it has the homotopy type of $S^{3} \times S^{5} \times \cdots \times S^{2 n-1}$. Its integral cohomology is an exterior algebra

$$
H^{*}(S U(n) ; Z)=\Lambda_{Z}\left[x_{3}, x_{5}, \ldots, x_{2 n-1}\right]
$$

where $\Lambda_{Z}\left[w_{5}, \ldots, w_{2 N-1}\right]$ is an exterior algebra on generators $w_{i}$ :

$$
\Lambda_{Z}\left[w_{5}, \ldots, w_{2 N-1}\right]=Z \oplus Z w_{5} \oplus Z w_{7} \oplus Z w_{9} \oplus Z w_{11} \oplus Z w_{5} w_{7} \oplus \cdots
$$

and, in particular, has no torsion. (In DeRham theory the $x_{i}$ may be represented by suitably normalized Maurer-Cartan forms.) It might therefore come as a surprise that 
torsion in the cohomology of $S U(n)$ (with other coefficient groups) is of central importance in understanding the geometry of WZW branes. The basic phenomenon can be understood in fairly elementary terms as follows.

Let us find smooth submanifolds of $S U(3)$ representing the Poincaré duals of $x_{3}$ and $x_{5}$. The Poincaré dual to $x_{5}$ is easily written down: it is an embedded $S U(2)$ subgroup of minimal Dynkin index. For definiteness, we will take it to be

$$
\left(\begin{array}{ll}
g & 0 \\
0 & 1
\end{array}\right) \quad g \in S U(2)
$$

This is a copy of $S^{3}$ and also represents a generator of $\pi_{3}(S U(3))$ and of $H_{3}(S U(3), Z)$.

The PD to $x_{3}$ is much more subtle. A nice representative is given by the submanifold $M_{5} \subset S U(3)$ of symmetric $S U(3)$ matrices. Let us make a few comments on this space.

First, $M_{5}$ intersects generic perturbations of the above $S U(2)$ subgroup in a single point. For example, if we take

$$
\left(\begin{array}{ccc}
c & 0 & s \\
0 & 1 & 0 \\
-s & 0 & c
\end{array}\right)\left(\begin{array}{cc}
g & 0 \\
0 & 1
\end{array}\right)
$$

as the representative of the generator of $H_{3}$, where $c^{2}+s^{2}=1$ and $s$ is nonzero, and $g \in S U(2)$, then the intersection with $M_{5}$ is readily seen to be the point

$$
\left(\begin{array}{ccc}
-c & 0 & s \\
0 & -1 & 0 \\
s & 0 & c
\end{array}\right)
$$

Since $M_{5}$ intersects a copy of $S^{3}$ in a single point we conclude that it is a generator of $H_{5}(S U(3) ; Z)$.

Next, $M_{5}$ is homeomorphic to the homogeneous space $S U(3) / S O(3)$ since (as one can show with a little bit of linear algebra) every symmetric $S U(3)$ matrix can be written in the form $g g^{t r}$ for some $g \in S U(3)$. 7 Thus, $M_{5}$ is a submanifold of $S U(3)$ but can also be regarded as a quotient of $S U(3)$.

7 First prove the analogous statement for $S U(2)$. Next, suppose $g$ is symmetric. It can be diagonalized $g=u D u^{-1}$. We get $\left(u^{t r} u\right) D=D\left(u^{t r} u\right)$. If the eigenvalues of $D$ are distinct $\left(u^{t r} u\right)=d$ is diagonal and we take $g_{0}=u d^{-1 / 2} D^{-1 / 2}$. If two eigenvalues coincide we are reduced to the $S U(2)$ case already considered. 
From the long exact sequence for fibrations we now get the exact sequence

$$
\cdots \rightarrow \pi_{i}(S U(3)) \rightarrow \pi_{i}\left(M_{5}\right) \rightarrow \pi_{i-1}(S O(3)) \rightarrow \pi_{i-1}(S U(3)) \rightarrow \cdots
$$

This shows that $M_{5}$ is simply connected, hence orientable, so $H^{0}\left(M_{5}, Z\right)=H^{5}\left(M_{5}, Z\right)=$ $\mathbf{Z}$, while $H^{4}\left(M_{5}, Z\right) \cong H_{1}\left(M_{5}, Z\right)=0$. This leaves unknown the middle cohomology groups. With a little more work one can deduce $H^{2}, H^{3}$ from (4.4) but an alternative argument, more useful for our purposes, can be given as follows.

We begin by noting that, over the reals, one can represent $x_{3}$ by the Maurer-Cartan form $x_{3}=\frac{1}{24 \cdot \pi^{2}} \operatorname{Tr}_{3}\left(g^{-1} d g\right)^{3}$, and this form vanishes identically on $M_{5}$ as a differential form. To prove this note that $x_{3}$ is left invariant and $M_{5}$ is an homogeneous space. Evaluation at 1 involves the trace of the commutator of two symmetric matrices against a third symmetric matrix, and hence vanishes. This fact suggests that $H^{3}\left(M_{5}, R\right)=0$, as indeed follows from the Thom isomorphism theorem. Therefore $H_{2}\left(M_{5}, R\right)$ and $H_{3}\left(M_{5}, R\right)$ are zero. Nevertheless, $H_{2}\left(M_{5}, Z\right)$ is nonzero as the following simple argument demonstrates. Consider a cycle $\Sigma_{2}$ in $M_{5}$ gotten by taking the intersection

$$
\Sigma_{2}:=S U(2) \cap M_{5}
$$

where $S U(2)$ is the subgroup (4.3) defined above. One readily checks that $\Sigma_{2}$ is the set of matrices

$$
\left(\begin{array}{ccc}
e^{i \phi} \cos \theta & i \sin \theta & 0 \\
i \sin \theta & e^{-i \phi} \cos \theta & 0 \\
0 & 0 & 1
\end{array}\right)
$$

and is hence a 2-sphere. This 2-sphere divides the $S U(2)$ subgroup of $S U(3)$ into hemispheres $D_{+} \cup D_{-}$. By symmetry $\int_{D_{+}} x_{3}=\int_{D_{-}} x_{3}=1 / 2$. We may now conclude that $\Sigma_{2}$ defines a nontrivial homology class in $M_{5}$. For, if not, we would fill it in with a 3-chain $\partial \mathcal{W}_{3}^{\prime} \subset M_{5}$ on which $x_{3}$ vanishes. But then $\Sigma_{3}=D_{+} \cup \mathcal{W}_{3}^{\prime}$ would be a three cycle in $S U(3)$ with $\int_{\Sigma_{3}} x_{3}=1 / 2$, but that is impossible for an integral class. This suggests that $H_{2}\left(M_{5}, Z\right)=Z_{2}$, as is indeed the case.

Using a little more technology (e.g. the Leray spectral sequence) one obtains the full cohomology groups of $M_{5}$ :

$$
\begin{aligned}
& H^{0}\left(M_{5}, Z\right)=Z \\
& H^{1}\left(M_{5}, Z\right)=0 \\
& H^{2}\left(M_{5}, Z\right)=0 \\
& H^{3}\left(M_{5}, Z\right)=Z_{2} \\
& H^{4}\left(M_{5}, Z\right)=0 \\
& H^{5}\left(M_{5}, Z\right)=Z
\end{aligned}
$$


By the universal coefficient theorems the cohomology with mod two coefficients is

$$
\begin{aligned}
& H^{0}\left(M_{5}, Z_{2}\right)=Z_{2} \\
& H^{1}\left(M_{5}, Z_{2}\right)=0 \\
& H^{2}\left(M_{5}, Z_{2}\right)=Z_{2} \\
& H^{3}\left(M_{5}, Z_{2}\right)=Z_{2} \\
& H^{4}\left(M_{5}, Z_{2}\right)=0 \\
& H^{5}\left(M_{5}, Z_{2}\right)=Z_{2}
\end{aligned}
$$

Now we come to a crucial point: The submanifold $M_{5}$ is not $\operatorname{Spin}^{c}$ ! We will now show this by establishing the equivalent statement that a certain characteristic class, $W_{3}\left(M_{5}\right) \in$ $H^{3}\left(M_{5} ; Z\right)$ is nonzero. In fact, we will show that $W_{3}\left(M_{5}\right)=\left.\left(x_{3}\right)\right|_{M_{5}} \neq 0$. (The remainder of this section assumes some knowledge of Steenrod squares. See [6], section 4.1 for relevant background.)

While the integral Steenrod operation $S q^{3}$ vanishes identically acting on the integral cohomology of $S U(3)$, the mod-two operation $S q^{2}$ does not, and indeed

$$
S q^{2}\left(r_{2}\left(x_{3}\right)\right)=r_{2}\left(x_{5}\right)
$$

where $r_{2}$ is reduction modulo two. 8 It follows at once that $Q=P D\left(x_{3}\right)$ is not Spin since, by definition, $S q^{2}(a)=a \cup \pi^{*}\left(w_{2}(\mathcal{N})\right)$ where $\pi: \mathcal{N} \rightarrow Q$ is the normal bundle in $S U(3)$. Moreover, $M_{5}$ is embedded in a spin (indeed, in a parallelizable) manifold so we can identify $w_{i}(\mathcal{N})=w_{i}\left(M_{5}\right)$. Next, note that $w_{2}\left(M_{5}\right) \in H^{2}\left(M_{5} ; \mathbf{Z}_{2}\right)$ is nonzero, and yet $H^{2}\left(M_{5} ; \mathbf{Z}\right)=0$, so $w_{2}$ does not have an integral lift and hence $W_{3}\left(M_{5}\right)$ is nonzero. In order to see the relation of $x_{3}$ and $W_{3}$ we combine the Wu formula $S q^{2}\left(r_{2}\left(x_{3}\right)\right)=w_{2} \cup r_{2}\left(x_{3}\right)$ with (4.9), and use the fact that $\left.x_{5}\right|_{Q}$ generates $H^{5}$, to conclude that $\left.\left(x_{3}\right)\right|_{M_{5}}$ is nonzero. Now, the Euler class of $Q=P D\left(x_{3}\right)$ is represented by $\left.x_{3}\right|_{Q}$, by Poincare duality. On the other hand, the Euler class of a real rank three oriented vector bundle is given by $W_{3}(\mathcal{N})=W_{3}\left(M_{5}\right)$.

In conclusion $W_{3}\left(M_{5}\right)=\left.x_{3}\right|_{M_{5}}$ is nonzero, and indeed generates $H^{3}\left(M_{5}, Z\right)$.

8 This equation is related by transgression to the equation $S q^{2}\left(r_{2}\left(c_{2}\right)\right)=r_{2}\left(c_{3}\right)$ in the cohomology of $B S U(n)$, a relation which also played an important role in [6]. 


\subsection{Branes in $S U(3)$ : Atiyah-Hirzebruch spectral sequence}

Let us now describe what information on $K_{H}^{*}(S U(3))$ for $H=k x_{3}$ can be gleaned from the AHSS. 9 Since $S q^{3}$ vanishes the differential is $d_{3}(\omega)=k x_{3} \cup \omega=H \cup \omega$. Therefore

$$
E_{3}^{j}=\left(\operatorname{ker} d_{3}: H^{j} \rightarrow H^{j+3}\right) /\left(\operatorname{Im} d_{3}: H^{j-3} \rightarrow H^{j}\right)
$$

is given by

$$
\begin{aligned}
& E_{3}^{j=3}=x_{3} Z / k x_{3} Z \cong Z_{k} \\
& E_{3}^{j=8}=x_{3} x_{5} Z / k x_{3} \wedge x_{5} Z \cong Z_{k}
\end{aligned}
$$

contributing to $K_{H}^{1}$ and $K_{H}^{0}$, respectively.

Now, for $S U(3)$ there could in principle be a further differential $d_{5}$. All we know is that this is a group homomorphism

$$
d_{5}: E_{3}^{3} \rightarrow E_{3}^{8}
$$

and therefore of the form $d_{5}(\omega)=s \omega$ for some integer $s$.

Then in the AHSS

$$
\begin{gathered}
K_{H}^{1}=\oplus_{\mathrm{j} \text { odd }} E_{\infty}^{j}=\oplus_{\mathrm{j} \text { odd }} E_{5}^{j} \cong \operatorname{ker}\left(d_{5}\right) \\
K_{H}^{0}=\oplus_{\mathrm{j} \text { even }} E_{\infty}^{j}=\oplus_{\mathrm{j} \text { even }} E_{5}^{j} \cong Z_{k} /\left(\operatorname{Im} d_{5}\right)
\end{gathered}
$$

There are no extension issues in passing from the associated graded to the full Ktheory because the associated graded is concentrated in a single cyclic group, for a given parity of the grading. Therefore we conclude

$$
K_{h}^{0}=K_{h}^{1}=Z_{k / s}
$$

for some $s$ which divides $k$. Since $d_{5}$ is unknown, $s$ is unknown. In fact, the results below show that $d_{5}$ is nontrivial.

One can also approach the problem using the six-term Meyer-Vietoris sequence for $K$-theory, but the most obvious decompositions lead only to (4.13).

9 GM would like to thank I. Brunner, G. Segal, and V. Schomerus, for discussions on such computations for $S U(n)$ groups, and their comparison to WZW models during August 2000. Very recently M. Hopkins has computed $K_{H}(S U(N))$. His result shows that the higher differentials are all nonzero. We describe his result, and our interpretation of it, in section 8 below. 


\subsection{Branes in $S U(3)$ : The physical computation}

Let us now consider the computation from the physical approach, as summarized by the two conditions (A) and (B) of the introduction.

We begin with the computation of $K_{H}^{1}$, which is a refinement of the odd cohomology. The Poincare dual of $x_{5}$ is an $S U(2)$ subgroup, and $H$ restricts to a nontorsion class. We cannot wrap branes on this cycle. The Poincare dual of $x_{3}$ can be represented by the submanifold $M_{5}$ of symmetric $S U(3)$ matrices, as discussed above. Implementing condition (A), the cancellation of global anomalies requires

$$
\left.[H]\right|_{\mathcal{W}}+W_{3}(\mathcal{W})=0
$$

where $\mathcal{W}$ is the brane worldvolume. As we have stressed, $W_{3}\left(M_{5}\right)$ is not zero so, if $H=k x_{3}$ then the condition (4.14) becomes

$$
r\left(k W_{3}+W_{3}\right)=0
$$

where $r$ is the number of times the worldvolume is wrapped. Thus we find that if $k$ is odd, there is no extra condition on $r$, while if $k$ is even then $r=0$ modulo 2. In the IIA theory in a spacetime with $X_{9}=S U(3) \times \mathbb{R}$ we should think of these as D6 branes. They wrap the 5 -cycle $\mathcal{W}$ in $S U(3)$ and the line.

Now let us implement condition (B) from the introduction. In this case D8 instantons wrapping $S U(3) \times R$ violate $\mathrm{D} 6$ brane number by $k$ units, as discussed above. We therefore conclude that the physical picture coincides with K-theory if

$$
K_{H=k x_{3}}^{1}(S U(3))= \begin{cases}\mathbf{Z} / k \mathbf{Z} & k \text { odd } \\ 2 \mathbf{Z} / k \mathbf{Z} & k \text { even }\end{cases}
$$

Let us now consider the computation of $K_{H}^{0}$, a refinement of the even cohomology of $S U(3)$. The even degree cohomology groups of $S U(3)$ are $H^{0}$ and $H^{8}$. The Poincare dual

of $1 \in H^{0}$ is all of $S U(3)$. Again, $H$ restricts to a nontorsion class and we cannot wrap branes on this cycle. Let us consider $H^{8}$. These correspond to D0-branes in IIA or $D 1$ branes wrapping $\mathbb{R}$ in $I I B$. In any case, there is no question of global anomalies, but we must worry about the effects of D-instantons. For definiteness we will focus on the IIA interpretation.

First, we have the familiar story: D2 instantons wrapping the $S U(2)$ subgroup violate $D 0$ charge by $k$ units. However, when $k$ is even there is another interesting instanton. As 
we have stressed, $M_{5}$ is not $\operatorname{Spin}^{c}$ so, if $k$ is even, we cannot wrap a D4 instanton on it. Let us do so anyway. Then there is a global anomaly, due to $W_{3}\left(M_{5}\right)$. We can cancel this global anomaly by adding a $D 2$ instanton that ends on a 2-cycle $\Sigma_{2} \subset M_{5}$ whose homology class $Q_{2}=\left[\Sigma_{2}\right]$ is Poincare dual (in $M_{5}$ ) to $W_{3}\left(M_{5}\right)$. Such a cycle is not a boundary in $M_{5}$, but can be bounded in $S U(3)$. Let us choose such a boundary $\mathcal{W}_{3} \subset S U(3)$ with $\partial \mathcal{W}_{3}=\Sigma_{2}$. This chain is anomaly free and constitutes a new type of D-instanton that appears when $k$ is even.

We now claim that the new D-instanton wrapping $\mathcal{W}_{3} \cup M_{5}$ in fact violates D0 charge by $k / 2$ units. This follows from the remarks around (4.5). Recall that the Maurer-Cartan form $x_{3} \sim \operatorname{Tr}\left(g^{-1} d g\right)^{3}$, vanishes identically on $M_{5}$ as a differential form. Moreover, the $\mathrm{PD}$ of $W_{3}\left(M_{5}\right)$ in $M_{5}$ can be represented by the cycle $\Sigma_{2} \cong S^{2}$ defined in (4.5). This 2-sphere divides the $S U(2)$ subgroup of $S U(3)$ into hemispheres $D_{+} \cup D_{-}$and we may choose $\mathcal{W}_{3}=D_{+}$in the above discussion. But such a D2-instanton violates D0 charge by $k / 2$ units! In this way we conclude that if K-theory is to match physical expectations then we must have

$$
K_{H}^{0}(S U(3))= \begin{cases}\mathbf{Z} / k \mathbf{Z} & k \text { odd } \\ \mathbf{Z} / \frac{k}{2} \mathbf{Z} & k \text { even }\end{cases}
$$

\section{Symmetry-Preserving branes in WZW models}

There is a large literature on D-branes in WZW models. A partial reference list includes [21-31]. Here we briefly review and extend the picture which has emerged, and comment on its relation to our discussion.

\subsection{Boundary states and conjugacy classes}

The WZW branes which are best understood are those that can be described by boundary states $|B\rangle\rangle \in \mathcal{H}^{\text {closed }}$ satisfying the boundary conditions

$$
\left.\left(J_{n}+\omega(\tilde{J})_{-n}\right)|B\rangle\right\rangle=0 \quad n \in \mathbf{Z}
$$

where $\omega$ is an automorphism of the affine Lie algebra. Here $J_{n}$ are the modes of the

left-moving currents and $\tilde{J}_{n}$ are the modes of the right-moving currents. Boundary states with $\omega=1$ are called "symmetry-preserving branes." (However, any choice of $\omega$ leaves an unbroken affine Lie algebra symmetry.) 
In what follows we will assume that we are working with the diagonal modular invariant

$$
\mathcal{H}^{\text {closed }}=\oplus_{\lambda \in P_{\kappa}^{+}} V_{\lambda} \otimes \tilde{V}_{\lambda^{*}}
$$

Here the sum is over the irreducible integrable representations $V_{\lambda}$ of the affine Lie algebra $\underline{\mathrm{g}}_{\kappa}$ based on a simple Lie algebra $\underline{\mathbf{g}}$. The notation $\tilde{V}$ indicates the right-moving component and $\kappa$ is the level of the current algebra.

One constructs boundary states with good sewing properties using the Cardy theory. To begin, we construct the character states, or "Ishibashi states." Choosing $\omega=1$, the character state $|\lambda\rangle\rangle_{I}$ is labelled by $\lambda \in P_{\kappa}^{+}$, and simply corresponds to the identity operator under the isomorphism $V_{\lambda} \otimes V_{\lambda}^{*} \cong \operatorname{Hom}\left(V_{\lambda}, V_{\lambda}\right)$. The Cardy states are expressed in terms of character states as

$$
\left.|\lambda\rangle\rangle_{C}=\sum_{\nu \in P_{\kappa}^{+}} \frac{S_{\lambda \nu}}{\sqrt{S_{0 \nu}}}|\nu\rangle\right\rangle_{I}
$$

where $S_{\lambda \nu}$ is the modular $S$-matrix and $\left.|\nu\rangle\right\rangle_{I}$ are the character states.

The Cardy states $|\lambda\rangle\rangle_{C}$ have a beautiful geometrical interpretation, valid at large $\kappa$ : They correspond to branes wrapping certain regular conjugacy classes of the group familiar from the theory of the Verlinde formula [22,23,24,25]. More precisely, these are the regular conjugacy class $\mathcal{O}_{\lambda, \kappa}$ of the element $\exp [2 \pi i(\lambda+\rho) \cdot H /(\kappa+h)] \in T$. For examples in level $\kappa$ $S U(2)$ theory the branes wrap conjugacy classes of trace $2 \cos \chi$ with $\chi=\pi(2 j+1) /(\kappa+2)$. For $S U(N)$ we label representations by Dynkin indices $\lambda=\sum_{i=1}^{N-1} a^{i} \lambda_{i}$, with $\sum a^{i} \leq \kappa$. The conjugacy class is the conjugacy class of

$$
\exp \frac{2 \pi i}{(\kappa+N)}(\lambda+\rho) \cdot H=\exp \left[\frac{2 \pi i}{(\kappa+N)} \sum_{l=1}^{N-1}\left(a^{l}+1\right)\left(\epsilon_{1}+\cdots+\epsilon_{l}-\frac{l}{N} \mathbf{1}\right)\right]
$$

where $\epsilon_{l}=e_{l l}$ is the $l^{t h}$ matrix unit along the diagonal.

The above geometrical picture may be justified by a straightforward generalization of the computation of appendix D of [14], see also [25]. We review the argument briefly. By the Peter-Weyl theorem an orthonormal basis for $L^{2}(G)$ in the unit Haar measure, for any compact group, is given by the matrix elements of unitary irreps: $\sqrt{d(\lambda)} D_{\mu_{L} \mu_{R}}^{\lambda}(g)$ where $d(\lambda)$ is the dimension of the representation $\lambda$. Therefore, a well-localized closed string state is

$$
|g\rangle=\sum_{\lambda \in P_{\kappa}^{+}, \mu_{L}, \mu_{R}} e^{-\epsilon c_{2}(\lambda) / \kappa} \sqrt{d(\lambda)} D_{\mu_{L} \mu_{R}}^{\lambda}(g)\left|\lambda, \mu_{L} \mu_{R}\right\rangle
$$


This is to be regarded as a state in $\mathcal{H}^{\text {closed }}$ made from vertex operators. The exponential regularizing factor is meant to suppress large representations, which correspond to "giant gravitons," rather than well-localized closed string states.

The modular $S$-matrix satisfies

$$
\frac{S_{\lambda, \nu}}{S_{\lambda, 0}}=\chi_{\lambda}\left(h_{\nu}\right)
$$

where, for compact, connected, simply-connected groups $G, h_{\nu}=\exp \left[2 \pi i \frac{\nu+\rho}{\kappa+h}\right]$. Therefore, we have

$$
\langle g \mid \hat{\lambda}\rangle\rangle_{C}=\sum_{\lambda} e^{-\epsilon c_{2}(\lambda) / \kappa} \sqrt{d(\lambda) S_{0 \lambda}} \chi_{\lambda}^{*}(g) \chi_{\lambda}\left(h_{\hat{\lambda}}\right)
$$

Moreover, for $\kappa \rightarrow \infty$

$$
\frac{S_{\lambda, 0}}{S_{00}} \rightarrow d(\lambda)
$$

Hence branes are localized 10 on the conjugacy classes $\mathcal{O}_{\lambda, \kappa}$ of the special elements $h_{\lambda}$.

Another way to justify the geometrical picture has been described by Felder et. al. in [25]. Among other things, these authors show that the algebra of boundary operators for boundary conditions $|\lambda\rangle\rangle_{C}$ becomes the algebra of functions on $G / T$ in the large $\kappa$ limit.

Since $\mathcal{H}^{\text {closed }}$ is a representation of $G_{L} \times G_{R}$, we may act on the boundary states to produce new states $\left.\rho_{L}\left(g_{L}\right) \rho_{R}\left(g_{R}\right)|\lambda\rangle\right\rangle_{C}$. It is clear from the above computation that, geometrically, these states correspond to branes wrapping the rotated classes $g_{L} \mathcal{O}_{\lambda, \kappa} g_{R}$. They satisfy boundary conditions of the type (5.1) where $\omega$ is an inner automorphism.

\subsection{Flux stabilization}

The above picture of D-branes raises a puzzle first pointed out, and resolved, in [27] in the case of $G=S U(2)$. The conjugacy classes $\mathcal{O}_{\lambda, \kappa}$ are homologically trivial in $G$. Since Dbranes carry tension one would expect the branes to be unstable to shrinking. Nevertheless, analysis of the open string channel (in the superstring) shows that the branes are stable.

As pointed out in [27], this paradox is resolved by recalling that the brane can carry a topologically nontrivial line bundle. In the present case there is a Chan-Paton line bundle $L$ (i.e. the brane is singly wrapped) which has $c_{1}(L)=\lambda+\rho$, where we identify $H^{2}(G / T ; Z)$

10 If we measure the position of the brane with fundamental string states then we can only determine the position to within a string length. Translated into uncertainty in the coordinates

in the maximal torus we find $\delta \psi \geq \frac{1}{\sqrt{\kappa}}$. One can also try to measure the branes using D-branes themselves, i.e., using the DKPS [32] limit. See [26] for further discussion of this point. 
with the weight lattice. Therefore $[F / 2 \pi]$ is nontrivial, and consequently terms of the form $\int_{\mathcal{O}} F \wedge * F$ increase as the radii of $\mathcal{O}$ decrease. This increases the energy of the brane, and balances the force due to the tension of the brane.

In appendix A we justify the above statements in detail by showing that the computation of [27] can be extended to higher rank groups.

\subsection{Blowing up D0 branes}

We will now review the "blowing up phenomenon" in the WZW model. We are reviewing here the work of [21,26, 27,29, 33].

Let us suppose the WZW model for $G$ a compact, connected, simply connected Lie group is a factor in the conformal field theory of a type II string compactification. Let us consider a system of $N$ D0 branes in the group. It seems clear that the Cardy state corresponding to the identity representation, $\lambda=0$, should be a D0 brane. We can see this from the shape computation, as well as from the open string spectrum which is that of a single D0 brane. It is reasonable to assign to this state D0 charge one. By putting together $N$ of these states it is obvious that we can have configurations with charge $N$. What is not so obvious is whether they can form a bound state. This problem was analyzed in the context of the Kondo model by Affleck and Ludwig [21]. What they found is the following. They started with $N$ D0 branes and they considered giving a small non-commutative vacuum expectation value to the D0 brane coordinates. The D0 brane coordinates are $N \times N$ matrices. Affleck and Ludwig chose these matrices to be $\epsilon S^{a}$ where $S^{a}$ is an $N \times N$ irreducible representation $\lambda$ of $G$. This amounts to introducing the boundary interaction $\int d t \epsilon S^{a} J^{a}(t, \sigma=0)$ in the string worldsheet. This interaction is marginally relevant [21]. At low energies the boundary field theory flows to a new boundary fixed point and therefore a new conformally invariant boundary condition. Affleck and Ludwig argued that the resulting fixed point is the Cardy state associated to $\lambda$. From an analysis of the DBI action one can estimate the time for this decay to be of order $\Delta T \sim \sqrt{\kappa}$ for natural initial conditions.

We summarize the Affleck-Ludwig argument here. Consider an open string stretching between one D0 brane and $N$ D0 branes. The boundary interaction described above results in a perturbation to the Sugawara Hamiltonian $\Delta H=\epsilon \sum_{n \in \mathbf{Z}} S^{a} J_{n}^{a}$ where $S^{a}$ represent the $N \times N$ generators of $\underline{\mathbf{g}}$ on $C^{N}$. Let us assume that this representation is $V_{\lambda} \cong C^{N}$

with $\lambda \in P_{+}$. Affleck and Ludwig observe that for the special value $\epsilon^{*}=\frac{2}{\kappa+h}$ the currents $\mathcal{J}_{n}^{a}:=J_{n}^{a}+S^{a}$ also form a Kac-Moody algebra which acts on $\left(V_{\hat{\lambda}=0} \otimes C^{N}\right)$, where $V_{\hat{\lambda}=0}$ 
is the $\hat{\lambda}=0$ representation of the $J^{a}$ current algebra. This product space is isomorphic to a highest weight representation with respect to the $\mathcal{J}_{n}^{a}$ current algebra. Indeed, the representation of $\mathcal{J}_{n}^{a}$ is isomorphic to $V_{\hat{\lambda}}$ where $\hat{\lambda}$ is the image of $\lambda$ in the level $\kappa$ Weyl alcove under the affine Weyl group. This last statement can be proved using the relation between the fusion product and the cabling of Wilson lines in 3d Chern-Simons theory 11 . This shows that the open string spectrum for a string stretched between a single D0 brane and the state resulting after the decay of $N$ D0 branes agrees with the expected open string spectrum between a D0 brane Cardy state and the Cardy state labeled by $\lambda$. Using a similar construction one can show that one can reproduce the open string spectrum between Cardy states labeled by $\lambda$ and $\lambda^{\prime}$.

This argument has simpler incarnations in some limits. In the large $\kappa$ limit and for a relatively small number of D0 branes $1 \ll N \ll \sqrt{\kappa}$ one can describe the process via the Myers effect [35], see [26,33] for further discussion. On the other hand, if $1 \ll \sqrt{\kappa} \ll$ $N \ll \kappa$, we can describe the resulting Cardy state in terms of a single D-brane wrapping a conjugacy class with a suitable $U(1)$ line bundle as in the flux-stabilization mechanism described above.

The Affleck-Ludwig argument is readily generalizable to string field theory, this is most obvious in BSFT.

\subsection{Worldsheet supersymmetry}

It is important for our considerations that we are working with the $\mathcal{N}=1$ supersymmetric WZW model. In this case a GSO projection can make the D-branes stable. Also, they become orientable and a D-brane charge can be defined.

The Kondo model renormalization group flows generalizes readily to the supersymmetric WZW case as we now show. The supersymmetric current algebra is [36, 37, 38]:

$$
\begin{aligned}
I_{a}(z) I_{b}(w) & \sim \frac{k \delta_{a b}}{(z-w)^{2}}+i f_{a b}{ }^{c} \frac{I_{c}(w)}{z-w}+\cdots \\
I_{a}(z) \psi_{b}(w) & \sim \frac{i f_{a b}^{c} \psi_{c}(w)}{z-w}+ \\
\psi_{a}(z) \psi_{b}(w) & \sim \frac{k \delta_{a b}}{(z-w)}+\cdots
\end{aligned}
$$

11 One studies the Hilbert space $\mathcal{H}$ of the Chern-Simons theory on a disk with a source in a representation $\lambda$ at a point $P$ in its interior [34]. $\mathcal{H}$ is determined as a product of $V_{\hat{\lambda}=0}$ on the boundary of the disk and $V_{\lambda}$ at $P$, or as $\mathcal{H}=V_{\hat{\lambda}}$ at the boundary. 
Note $\left\{Q, \psi_{a}\right\}=I_{a}$ and $\left\{Q, I_{a}\right\} \sim \partial \psi_{a}$. As is well-known, one can define decoupled currents $J_{a}=I_{a}+\frac{i}{2 k} f_{a b c} \psi_{b} \psi_{c}$ which satisfy a current algebra with level

$$
\kappa=k-h
$$

where $h$ is the dual Coxeter number. The level that should be used in the K-theory analysis in section 4 is $k$.

In the Kondo model we use the supersymmetric perturbation $\int d \tau S^{a} I_{a}(\sigma=0, \tau)$. It is easy to think about the flow as deforming the supercharge by $S^{a} \psi^{a}$. At the infrared fixed point we can apply the Affleck-Ludwig trick again to $J_{a} \rightarrow J_{a}+S^{a}$. Therefore, the fermionic part of the boundary state is unchanged and the flow is effectively only in the bosonic part of the state, although the fermions and the bosons do not decouple along the renormalization group trajectory.

\subsection{Assigning D0 charge to the symmetry-preserving branes}

We now consider the problem of assigning a K-theory class to the explicit conformal field theory boundary states discussed in the previous subsections.

There are two ways to associate a D0 charge to a collection of D0-branes in the level $k$ WZW theory. First, one can simply compute the integral $\int_{\mathcal{O}_{\lambda, k}} e^{F+B}$ appearing the the standard WZ coupling of D-branes. This leads to quantum dimensions, a puzzling result, which we will not try to address in this paper.

A more straightforward approach is simply to invoke the blowing-up phenomenon described in section 5.3. It is natural to try to define the D0 charge of the Cardy state labeled by $\lambda$ to be the dimension of the representation $\lambda$, denoted $d(\lambda)$. Note, however, that at fixed $k$ there are only a finite number of integrable representations. We therefore assume that the D0 charge is torsion and is given by $d(\lambda) \bmod n(\kappa, G)$ where $n(\kappa, G)$ is an integer depending on $\kappa$ and $G$. It is clear from the existence of D2 instantons wrapping the $S U(2)$ subgroup of $G$ that the D0 charge will be $k$-torsion. (Recall that $\kappa$ and $k$ are related as in (5.10).) However, as we saw in section 4 , there can be more subtle instantons arising from branes wrapping higher dimensional homologically nontrivial cycles which nevertheless violate D0 charge. We will now describe the result for $n(\kappa, S U(N))$.

To begin, let us consider the case of $G=S U(2)$. The classes $\mathcal{O}_{\lambda, \kappa}$ correspond to $g=\exp [i \chi \hat{n} \cdot \vec{\sigma}]$ for $\chi=\pi l /(\kappa+2)$ where $l=1, \ldots, \kappa+1$. Together with $l=0$, corresponding to no D0 branes at all, this suggests a charge group $Z_{\kappa+2}=Z_{k}$. 
Unfortunately, this simple argument does not generalize to higher rank groups. A formula for $n(\kappa, S U(N))$ was proposed by Fredenhagen and Schomerus [33] based on the renormalization group flows in the Kondo model [21], as reviewed above. We will now offer an alternative derivation. Our argument is also based on the validity of the blowing-up hypothesis. Our argument is very similar to an argument given in 29].

We will use the left $\times$ right rotational $G \times G$ symmetry mentioned at the end of section 5.1. In particular, we consider the simple action of rotating the conjugacy class by the left action of an element of the center $z \in Z(G)$.

Now, the center of $G$ may be identified with a subgroup of the group of automorphisms of the extended Dynkin diagram of $G$ [39,40]. The center of $G$ rotates orbits, while the automorphisms of the extended Dynkin diagram act on the integrable level $\kappa$ representations. The two actions are related by $z \mathcal{O}_{\lambda, \kappa}=\mathcal{O}_{\lambda^{\prime}, \kappa}$. In particular, for $S U(N)$ one easily checks the identity

$$
\exp \left[\frac{2 \pi i}{\kappa+N}\left(\lambda^{\prime}+\rho\right) \cdot H\right]=z \sigma \exp \left[\frac{2 \pi i}{\kappa+N}(\lambda+\rho) \cdot H\right] \sigma^{-1}
$$

Here $z=e^{-\frac{2 \pi i}{N}} 1_{N}$ generates $Z(S U(N))$, while $\sigma$ is a permutation matrix taking $\sigma \epsilon_{i} \sigma^{-1}=$ $\epsilon_{i+1}$ (corresponding to the Coxeter element of the Weyl group). The transformation $\lambda \rightarrow \lambda^{\prime}$ acts on Dynkin indices as

$$
\lambda=\sum_{i=1}^{N-1} a^{i} \lambda_{i} \rightarrow\left(\kappa-\sum_{i=1}^{N-1} a^{i}\right) \lambda_{1}+a^{1} \lambda_{2}+\cdots+a^{N-2} \lambda_{N-1} .
$$

This is the standard action of the $Z_{N}$ outer automorphism of $\widehat{S U(N)}$ on weight vectors and it generates the spectral flow transformations.

Now, if one considers the action of $z \in Z(G)$ on the Cardy states, one finds that $z|\lambda\rangle\rangle_{C}$ corresponds to the rotated conjugacy class $z \mathcal{O}_{\lambda, \kappa}$, as well as to the state $\left.\left|\lambda^{\prime}\right\rangle\right\rangle_{C}$. To prove this we use the property of the modular $S$-matrix [39,40

$$
S_{\lambda^{\prime}, \mu}=S_{\lambda, \mu} e^{-2 \pi i\left(\lambda_{1}, \mu\right)}
$$

where $\lambda_{1}$ is the fundamental representation. The phase on the right hand side of (5.13) is precisely the action of rotation of left-movers by $e^{-\frac{2 \pi i}{N}}$ when acting on the Ishibashi state $|\mu\rangle\rangle_{I}$.

Now, a rigid rotation of a brane cannot change its D0 charge. Therefore, we require

$$
d\left(\lambda^{\prime}\right)=(-1)^{N-1} d(\lambda) \bmod n(\kappa, G) .
$$


The origin of the relative sign is the following: The orientation of a brane wrapping a conjugacy class rotated from $\mathcal{O}_{\lambda, \kappa}$ by $z$ and a brane wrapping a conjugacy class $\mathcal{O}_{\lambda^{\prime}, \kappa}$ resulting from blowing up D0 branes might differ. Since the Coxeter element is a product of $(-1)^{N-1}$ reflections the relative orientation beteen the rotated and the blown-up brane differs by this factor.

It is not difficult to show that if two generic conjugacy classes in $G=S U(N)$ are related by rotation $\mathcal{O}=\mathcal{O}^{\prime} g$ then $g \in Z(G)$, and hence (5.14) (and its spectral flow descendents) are the only conditions we should impose.

The equation (5.14) is a nontrivial constraint on $n(\kappa, G)$. For $S U(2)$ we have $d(j)=$ $2 j+1$ so

$$
2\left(\frac{\kappa}{2}-j\right)+1=-(2 j+1) \bmod n(\kappa)
$$

leading to $n(\kappa)=\kappa+2=k$. For $S U(3)$ we have $d(\lambda)=\frac{1}{2}(a+1)(b+1)(a+b+2)$ for $\lambda=(a, b)$, while and $\lambda^{\prime}=(\kappa-a-b, a)$. Then $d(\lambda)=d\left(\lambda^{\prime}\right) \bmod n(\kappa)$ determines $n(\kappa)=\kappa+3=k$ for $\kappa$ even and $n(\kappa)=(\kappa+3) / 2=k / 2$ for $\kappa$ odd. For general $N$ we reproduce the answer of Fredenhagen and Schomerus:

$$
n(\kappa, S U(N))=\operatorname{gcd}\left\{a_{i}:=d\left(\kappa \lambda_{1}+\lambda_{i}\right)\right\}=\frac{\kappa+N}{\operatorname{gcd}(\kappa+N, \operatorname{lcm}(1,2, \ldots, N-1))}
$$

\section{Remarks:}

1. The detailed connection to the argument in [33] is the following. They consider the representation $\kappa \lambda_{1}$. This has $S U(N)$ fusion rules

$$
\kappa \lambda_{1} \times \lambda_{i}=\left(\kappa \lambda_{1}+\lambda_{i}\right) \oplus\left((\kappa-1) \lambda_{1}+\lambda_{i+1}\right)
$$

and $\widehat{S U(N)})_{\kappa}$ fusion rules

$$
\kappa \lambda_{1} \times \lambda_{i}=\left((\kappa-1) \lambda_{1}+\lambda_{i+1}\right)
$$

If one identifies the "charge group" as $\mathbf{Z}_{x}$ where $x$ is the smallest integer such that

$$
d\left(\kappa \lambda_{1}\right) d\left(\lambda_{i}\right)=d\left(\left((\kappa-1) \lambda_{1}+\lambda_{i+1}\right)\right) \bmod x
$$

then one finds [33], $x=\operatorname{gcd}\left(a_{i}\right)$, with $a_{i}=d\left(\kappa \lambda_{1}+\lambda_{i}\right)$. To connect to our argument one proves the identity

$$
\sum_{i=1}^{N-1}(-1)^{i} a_{i}+(-1)^{N} d\left(\kappa \lambda_{1}\right)=-1
$$


by using Weyl's product formula. It thus follows that $d\left(\kappa \lambda_{1}\right)=(-1)^{N-1} \bmod x$, so (5.19) becomes (5.14).

2. It is important to realize that in a charge group not all charges need be realized, and some charges might be realized more than once. The reader need only compute the charges $d(\lambda) \bmod n(\kappa, S U(3))$ for $\kappa=2$ to see some examples.

\section{WZW branes from outer automorphisms}

The branes defined by the boundary condition (5.1) have been investigated in [25, 33]. These branes wrap twinned conjugacy classes associated with the automorphism $\omega$. Here we comment on these branes, mainly to facilitate comparison with our computation of $K_{H}^{1}(S U(3))$ in section 8 .

\subsection{The shape of the symmetry-preserving boundary states for outer automorphisms}

Let us focus on the case of the outer automorphism $\omega_{c}$ of $S U(3)$ given by complex conjugation. By (5.1) the representation of the zeromode algebra must be such that $\lambda_{L}=$ $\lambda_{R}$. Since we are working with the diagonal modular invariant theory, the weights must also be related by $\lambda_{R}=\lambda_{L}^{*}$ to exist in the closed string spectrum. Therefore the allowed representations $\lambda$ are the symmetric weights with Dynkin labels $(n, n), n=0,1, \ldots,[\kappa / 2]$.

According to [30,25] there is a natural basis of solutions to (5.1) given by "twinned character states" (or Ishibashi states) $|\lambda\rangle\rangle_{I}^{\omega_{c}}$ which are defined such that

$$
\begin{gathered}
\stackrel{\omega}{I}_{I}\left\langle\left\langle(b, b)\left|q_{c}^{\frac{1}{2}(H+\tilde{H})}\right|(a, a)\right\rangle\right\rangle_{I}^{\omega}=\delta_{a, b} \chi_{(b, b)}\left(q_{c}\right) \\
{ }_{I}\left\langle\left\langle(b, b)\left|q_{c}^{\frac{1}{2}(H+\tilde{H})}\right|(a, a)\right\rangle\right\rangle_{I}^{\omega}=\delta_{a, b} \chi_{(b, b)}^{\omega}\left(q_{c}\right)
\end{gathered}
$$

where $\chi^{\omega}$ are the "twinned characters" of [31]. For explicit expressions, see [31,30], and references therein.

The corresponding "Cardy" boundary states with good sewing properties are obtained using a modular transformation matrix $S_{a b}^{\omega}($ for $\lambda=(a, a), \mu=(b, b))$ given by

$$
S_{a b}^{\omega}=\frac{2}{\sqrt{\kappa+3}} \sin \left[2 \pi \frac{(a+1)(b+1)}{(\kappa+3)}\right]
$$

(For $\kappa$ odd this is the S-matrix of an $\mathrm{SU}(2)$ theory at level $[\kappa / 2]$.) Explicitly,

$$
\left.|(a, a)\rangle\rangle_{C}^{\omega}=\sum_{b} \frac{S_{a b}^{\omega}}{\sqrt{S_{0,(b, b)}}}|(b, b)\rangle\right\rangle_{I}^{\omega}
$$


We can now measure the shape of the branes $|(a, a)\rangle\rangle_{C}^{\omega}$ by taking the overlap with a well-localized closed string state. We parametrize elements of $S U(3)$ as

$$
g=h\left(\begin{array}{ccc}
\cos \theta & \sin \theta & 0 \\
-\sin \theta & \cos \theta & 0 \\
0 & 0 & 1
\end{array}\right) h^{t r}
$$

then we have the overlap

$$
\langle g \mid(a, a)\rangle\rangle_{I}^{\omega_{c}}=\frac{\sin 2 \phi(a+1)}{\sin 2 \phi}
$$

where we have defined an angle

$$
1+2 \cos \phi=-\cos \theta \quad \Rightarrow \quad \cos \phi=-\left(\cos \frac{1}{2} \theta\right)^{2}
$$

The necessity for this change of angles lies in the difference between a diagram automorphism and the complex conjugation automorphism.

At large $\kappa$ we find

$$
\begin{aligned}
\langle g \mid(\hat{a}, \hat{a})\rangle\rangle_{C}^{\omega} & \sim \frac{\kappa^{3}}{\sin 2 \phi} \sum_{b=0}^{[\kappa / 2]} \sin ((b+1) \phi) \sin \left((b+1) \hat{\psi}_{a}\right) \\
& \sim \frac{\kappa^{3}}{\sin 2 \phi} \delta_{\text {periodic }}\left(2 \phi-\hat{\psi}_{a}\right)
\end{aligned}
$$

where $\hat{\psi}_{a}=2 \pi(\hat{a}+1) /(\kappa+3)$ and we have dropped overall numerical factors.

Thus, geometrically, the branes (6.3) are branes wrapping the twinned conjugacy classes

$$
\mathcal{O}^{\omega}(t):=\left\{h t h^{-1, *}: h \in S U(3)\right\}=\left\{h t h^{t r}: h \in S U(3)\right\}
$$

for $t \in T_{2}^{\omega}$, where we have the maximal torus

$$
T_{2}^{\omega}=\left\{\left(\begin{array}{ccc}
\cos \theta & \sin \theta & 0 \\
-\sin \theta & \cos \theta & 0 \\
0 & 0 & 1
\end{array}\right)\right\}
$$

Note that the manifold $M_{5}$ of section 4.2 is just the twinned conjugacy class of the identity. From $(6.7)$ we see that the branes corresponding to the boundary states $|(a, a)\rangle\rangle_{C}^{\omega_{c}}$ wrap twinned conjugacy classes localized at discrete values of $\theta$, whose values can be read off from (6.7) and (6.6). For generic $\theta$ the twinned conjugacy classes may be identified with the 7-dimensional homogeneous spaces of the form $S U(3) / U(1)$. They are $S^{2}$ bundles over the twinned conjugacy class of the identity, $M_{5} .12$

12 It can be useful to view these as ordinary conjugacy classes in the disconnected component of $\mathbf{Z}_{2} \ltimes S U(N)$. See [41] for several relevant results on these classes. 


\subsection{Spectrum, wrapping number, and gauge bundle on the twinned branes}

The cohomology groups in (4.7) show that we cannot put topologically nontrivial Chan-Paton line bundles on $M_{5}$, hence we do not expect any further superselection sectors for branes in $S U(3)$.

Using the above boundary states and passing to the open string channel we can examine the spectrum of the "twinned brane." Unitarity of $S_{a b}^{\omega}$ shows that the unit operator appears once.

Let us consider the masses and wrapping numbers of the twinned branes. From conformal field theory we can compute the mass of the twinned brane relative to a D0 brane:

$$
\frac{S_{a 0}^{\omega}}{S_{00}}=\sqrt{3} \frac{(\kappa+3)^{7 / 2}}{(2 \pi)^{3}} \sin \left(2 \pi \frac{a+1}{\kappa+3}\right)
$$

which behaves correctly as a function of $\kappa$ in the sense that for $a \ll \kappa$ we have a 5 dimensional object but for $a \sim \kappa$ we have a 7-dimensional object.

On the other hand, using equation B.5 of the appendix we see that an $L$-times wrapped brane on $M_{5}$ has a mass

$$
L \cdot\left(\frac{\kappa}{2}\right)^{5 / 2} 4 \sqrt{\frac{3}{2}}(2 \pi)^{3} \frac{1}{(2 \pi)^{5}}=\frac{L}{2} \kappa^{5 / 2} \sqrt{3} \frac{1}{(2 \pi)^{2}}
$$

where the last factor in the right hand side is the D-brane tension. We thus conclude that, for $a \ll \kappa$ the wrapping number is

$$
L=2 a+2,
$$

and is always even! On the other hand, for $a \approx \kappa / 2$ we must rewrite $\sin \left(2 \pi \frac{a+1}{\kappa+3}\right)=$ $\sin \left(\pi \frac{\kappa-2 a+1}{\kappa+3}\right)$ from which we conclude

$$
L=\kappa-2 a+1 \quad a \approx \kappa / 2
$$

If $\kappa$ is even then we can set $a=\kappa / 2-n, n=0,1,2$ and the wrapping number is $2 n+1$, and always odd. If $\kappa$ is odd then $a=\frac{\kappa-1}{2}-n$ and the wrapping number is $L=2 n+2$ and always even.

Let us now compare these results on wrapping number with the considerations of section 4. We start with the bosonic string. Here a necessary condition for being able to wrap branes is equation (1.1) without the $W_{3}(\mathcal{W})$ term $[4, \mathbb{8}]$. In our case $\left.[H]\right|_{\mathcal{W}}=0$ in $H^{3}(\mathcal{W}, Z)$ translates to $\kappa L \in 2 Z$. Therefore, for $\kappa$ even all values of $L$ are allowed, while 
for $\kappa$ odd only even values of $L$ are consistent. This is exactly the result found above in the conformal field theory.

Turning to the superstring, we must recall from (5.10) that $k=\kappa+3$ determines the cohomology class of $H=d B$. This shows that the parity of $\kappa$ (used here) is opposite to $k$. Bearing this in mind and adding back the $W_{3}(\mathcal{W})$ term, which is not present in the bosonic string, we again find the condition $\kappa L \in 2 Z$. Again, we see a perfect match between results of the conformal field theory and the implications of the topology discussed in section 4 .

The $\mathrm{K}$ theory considerations are based on anomaly cancellation and the requirement

that $\exp \left[i \int_{\Sigma} B\right]$ must be well defined for worldsheets ending on the brane. Even though the anomaly can only be seen at the level of torsion cohomology classes, the boundary state "knows" about it. We find this somewhat remarkable.

\section{Parafermionic branes}

Boundary conditions of the type (5.1) construct rather special branes, preserving a full current algebra symmetry. There are many other branes in WZW theory one can construct by generalizing the methods of [15]. (See also the works of Fuchs and Schweigert et. al.) Here we will only sketch the theory of these branes, leaving a detailed analysis for another occasion.

Given the WZW chiral algebra $\mathcal{A}(G)$ it is natural to choose a maximal torus $T \subset G$ and form the parafermionic chiral algebra $\mathcal{A}(G / T)$. This may be defined by the coset construction with respect to the level $\kappa$ torus chiral algebra $\mathcal{A}(T)$. The latter is obtained as follows. We begin by bosonizing the currents in the maximal torus:

$$
H^{i}(z)=-i \sqrt{\kappa} \partial_{z} \phi^{i} \quad i=1, \ldots, r
$$

where $r$ is the rank of $G$. The scalar $\phi$ has periodicity

$$
\phi \sim \phi+2 \pi \sqrt{\kappa} Q
$$

where $Q$ is the root lattice of $G$. The full chiral algebra $\mathcal{A}(T)$ is extended by the vertex operators

$$
\exp [i \sqrt{\kappa} \alpha \cdot \phi] \quad \alpha \in Q
$$

The representations of $\mathcal{A}(T)$ are generated by

$$
\exp \left[i \frac{1}{\sqrt{\kappa}} \mu \cdot \phi\right] \quad \mu \in P
$$


where $P$ is the weight lattice of $G$. The characters of these representations are level $\kappa$ theta functions $\Theta_{\kappa, \mu} / \eta^{r}$. The label $\mu$ on representations is subject to $\mu \sim \mu+\kappa Q$. Thus the representations are labelled by $P / \kappa Q$. For $S U(N)$ this has cardinality $N \kappa^{r}$.

There is now a straightforward procedure for producing the $\mathcal{A}(G) / \mathcal{A}(T):=\mathcal{A}(G / T)$ coset model. The characters are obtained from string functions defined by:

$$
\chi_{\Lambda}(\tau, z)=\sum_{\mu \in P / \kappa Q} c_{\Lambda, \mu}(\tau) \frac{\Theta_{\kappa, \mu}(\tau, z)}{\eta^{r}(\tau)}
$$

For $G=S U(N)$, the parafermionic representations are subject to a $Z_{N}$ selection rule. $\Lambda-\mu \in Q$, as well as a $Z_{N}$ identification from the outer automorphism of the Lie algebra $(\Lambda, \mu) \sim\left(\Lambda^{\prime}, \mu+\kappa \lambda_{1}\right)$ where $\Lambda \rightarrow \Lambda^{\prime}$ is defined in (5.12).

The finite group $\Gamma=Q / \kappa Q$ acts as a group of global symmetries on both the $G / T$ theory and the $T$ theory, with $\beta \in Q / \kappa Q$ acting as

$$
\begin{aligned}
\Psi_{\Lambda, \mu} & \rightarrow e^{2 \pi i \mu \cdot \beta / \kappa} \Psi_{\Lambda, \mu} \\
\Psi_{\mu} & \rightarrow e^{-2 \pi i \mu \cdot \beta / \kappa} \Psi_{\mu}
\end{aligned}
$$

If we take the orbifold chiral algebra with this action we obtain the original $G$ WZW chiral algebra, $(\mathcal{A}(G / T) \otimes \mathcal{A}(T)) / \Gamma \cong \mathcal{A}(G)$. Therefore one can form branes in the $G$ WZW theory using branes from the parafermionic theory. The advantage of this viewpoint is that we can construct new branes which do not have simple boundary conditions in terms of the currents.13

For example, the symmetry-preserving branes are of the form

$$
\left.\left.|\Lambda\rangle\rangle_{C}^{G}=\sqrt{\frac{1}{|\Gamma|}} \sum_{\mu \in P / \kappa Q}|\Lambda, \mu\rangle\right\rangle_{C}|\mu\rangle\right\rangle_{C}
$$

However, now we can form the "B-type branes" in the language of [15]. For example, one can now perform T-duality with respect to the various $U(1)$ currents in the $\mathcal{A}(T)$ theory. For example, let $G=S U(N+1)$ and consider the embedding $S U(N) \times U(1) \hookrightarrow S U(N+1)$ where $U(1)$ corresponds to the generator $H^{N}=\frac{1}{\sqrt{N(N+1)}} \operatorname{Diag}\{1, \ldots, 1,-N\}$ of the Lie algebra. Performing $T$-duality with respect to the current $H^{N}(z)$ we get

$$
\left.\left.|\Lambda\rangle\rangle_{C}^{\prime}, G=\sqrt{\frac{1}{|\Gamma|}} \sum_{\mu \in P / \kappa Q}|\Lambda, \mu\rangle\right\rangle_{C}|\mu\rangle\right\rangle_{C}^{\prime}
$$

13 It might be interesting to understand whether these boundary fixed point theories are of use in the multi-channel Kondo problem. 
here $|\mu\rangle\rangle_{C}^{\prime}$ is a state in the $\mathcal{A}(T)$ theory. It is $A$-type with respect to the first $(N-1)$ currents and $B$-type with respect to $H^{N}(z)$, and only depends on $\mu-\left(\alpha_{N} \cdot \mu\right) \lambda_{N}$.

To see that (7.8) is a qualitatively new brane we will sketch the shape computation, showing that it can be a $(2 N+1)$-dimensional object in $G$. The state (7.8) satisfies boundary conditions:

$$
\begin{aligned}
\left.\left(J_{n}^{a}+\tilde{J}_{-n}^{a}\right)|B\rangle\right\rangle & =0 \quad a \in \operatorname{su}(N) \\
\left.\left(H_{n}^{N}-\tilde{H}_{-n}^{N}\right)|B\rangle\right\rangle & =0
\end{aligned}
$$

(but does not satisfy simple boundary conditions with respect to the remaining currents). Let us now work infinitesimally and consider the "shape computation" for group elements

$g=1+\sum_{j=1}^{N}\left(z^{j} e_{N+1, j}-\bar{z}^{j} e_{j, N+1}\right)+\cdots$ where $z^{j}$ are complex numbers and $e_{i j}$ are matrix units. We would like to show that the overlap with the Cardy states $\langle g| \hat{\lambda}, \hat{\mu}=$ $0\rangle\rangle_{C}^{B}$ is independent of $z^{j}$. To show this, note that the zeromode part of the "B-branes" necessarily have $\left.\left.H_{0}^{N}|B\rangle\right\rangle=\tilde{H}_{0}^{N}|B\rangle\right\rangle=0$. Now, in any $S U(N+1)$ representation $\Lambda$ if $H^{N}\left|\mu_{L}\right\rangle=H^{N}\left|\mu_{R}\right\rangle=0$ then

$$
\left(\alpha^{(N)}+\alpha^{(j)}\right)_{N}\left\langle\mu_{L}\left|\rho_{\Lambda}\left(e_{j, N+1}\right)\right| \mu_{R}\right\rangle=\left\langle\mu_{L}\left|\rho_{\Lambda}\left(\left[H^{N}, e_{j, N+1}\right]\right)\right| \mu_{R}\right\rangle=0
$$

This shows that $\langle g \mid \Lambda\rangle\rangle_{C}^{\prime}, G$ is independent of $z^{j}$, to first order in $z^{j}$. It is important to include higher order terms in $z^{j}$, but we will not do this here.

We conjecture that, at least for some $\Lambda$, the brane described by (7.8) wraps a homologically nontrivial cycle in $S U(N+1)$ transverse to the $S U(N)$ subgroup. This cycle is explicitly the union of conjugacy classes:

$$
w_{2 N+1}=\cup_{g \in S U(N+1), z \in U(1)} g \operatorname{Diag}\left\{z^{-1 / N}, \ldots, z^{-1 / N}, z^{1-1 / N}\right\} g^{-1}
$$

This is also the set of unitary matrices of the form $g_{i j}=z^{-1 / N}\left(\delta_{i j}+(z-1) v_{i} \bar{v}_{j}\right)$ where $v_{i}$ is a unit vector in $C^{N+1}$ and $z \in U(1)$. It is the image in $S U(N+1)$ of a continuous map $S^{1} \times C P^{N} \rightarrow S U(N+1)$ which factors through the unreduced suspension.

\section{Comparison with K-theory}

\subsection{The result of M. Hopkins}

Here we describe the result of a recent computation of M. Hopkins. As we have mentioned, $S U(N)$ is rationally a product of odd spheres $S^{3} \times S^{5} \times \cdots \times S^{2 N-1}$. Branes cannot wrap a homology cycle with $S^{3}$ as a factor, because of the $H$-flux, but they can 
wrap all other cycles (sometimes, we require finite multiplicity, as we have seen). This picture of D-branes in $S U(N)$ at level $k$ suggests a charge group

$$
\left(Z / d_{k, N} Z\right) \otimes \Lambda_{Z}\left[w_{5}, \ldots, w_{2 N-1}\right]
$$

More explicitly, the charge group is

$$
Z_{d_{k, N}} \oplus Z_{d_{k, N}} w_{5} \oplus Z_{d_{k, N}} w_{7} \oplus Z_{d_{k, N}} w_{9} \oplus Z_{d_{k, N}} w_{11} \oplus Z_{d_{k, N}} w_{5} w_{7} \oplus \cdots
$$

One can identify the $w_{i}$ with branes wrapping the primitive homology cycles of dimension $i$, generating the homology of $S U(N)$. The result (8.1) for the $K_{H}$-homology of $S U(N)$ has, in fact, been obtained recently by M. Hopkins [42], using a standard cell decomposition of $S U(N)$ [43], together with the Meyer-Vietoris sequence. The cells are given by the construction in (7.11).

The computation of Hopkins leads to the formula

$$
d_{k, N}=\operatorname{gcd}\left[\left(\begin{array}{l}
k \\
1
\end{array}\right),\left(\begin{array}{l}
k \\
2
\end{array}\right), \ldots,\left(\begin{array}{c}
k \\
N-1
\end{array}\right)\right] .
$$

In appendix $\mathrm{C}$ we show that this expression coincides with the expression found by Fredenhagen and Schomerus, remembering that $k=\kappa+N$

$$
d_{k, N}=n(\kappa, S U(N))
$$

In order to compare with the K-theory formalism we must use the supersymmetric $S U(N)$ WZW model at the level $k$, defined by the coupling to the $B$ field in the worldsheet Lagrangian with supersymmetric fermions.

\subsection{Comparison for $S U(3)$}

The result of Hopkins confirms our computation of $K_{H}^{*}(S U(3))$ in section 4.4. Moreover we have constructed branes corresponding the different K-theory classes in this case.

In particular, using the results of section 6 , we can give a picture for the branes contributing to the classes in $K_{H}^{1}(S U(3))$. These are branes wrapping the twinned conjugacy classes $\mathcal{O}^{\omega}$. As we noted above, these are $S^{2}$ bundles over $M_{5}$. Since $S U(2)$ intersects $M_{5}$ transversally we can interpret these branes as wrapping $M_{5}$, but blown-up along the transverse $S U(2)$ into a bundle of $S^{2}$ 's over $M_{5}$. 


\subsection{Comparison for $S U(N)$}

We have not generalized the computation of section 4 to $S U(N)$, but this is in principle possible.

The symmetry-preserving branes described in section 5 account for the first term in (8.2). Branes associated to the outer automorphism of $S U(N)$, wrapping twinned conjugacy classes, might carry other K-theory charges but clearly are not numerous enough to fill out the full set of K-theory classes predicted by (8.1).

We conjecture that applying T-dualities to the parafermionic branes in $S U(N)$ will lead to representatives of all the K-theory classes.

\section{Discussion}

A potentially fruitful open problem is that of formulating a definition of the group of "charges" of (all) branes and fluxes allowed in string theory and M-theory. This charge group would help to distinguish the different homotopy classes of collections of branes and fluxes. By "homotopy classes" we mean we identify brane configurations related to one another by continuous deformations of parameters, e.g., continuously deforming the position of a D-brane, or the gauge fields on a D-brane. etc.

The present paper offers one approach to this problem. In broad terms the group of components should be the group of homotopy classes of anomaly-free brane configurations modulo equivalence relations imposed by dynamically allowed effects.

This "physical definition" of a group of components cannot fail to be right, but it is also not very precise or useful. We can make it more precise by focusing on D-branes at weak coupling. Then we have seen in this paper that the charge group can be viewed as a "quotient" of classes of anomaly-free configurations of branes by identifications by Dinstanton effects. This philosophy offers, perhaps, an alternative route to the definition of $\mathrm{K}$-theory, or of the appropriate notion which will replace K-theory when one is not working at zero string coupling. It is possible that all the essential physical effects determining Dbrane charges have already been discovered. If this is so, then this approach to a precise mathematical framework for D-brane charges has a reasonable chance of succeeding. 


\section{Acknowledgements}

GM would like to thank I. Brunner, E. Diaconescu, D. Freed, M. Hopkins, P. Landweber, D. Morrison, G. Segal, and E. Witten for many important discussions on the topology and K-theoretic interpretation of D-branes. We thank D. Freed for comments on the draft.

GM and JM would like to thank the ITP at Santa Barbara for hospitality during the writing of part of this manuscript. This research was supported in part by the National Science Foundation under Grant No. PHY99-07949 to the ITP by DOE grant DE-FG0296ER40949 to Rutgers.

JM and NS are supported in part by DOE grants \#DE-FG02-90ER40542, \#DEFGO2-91ER40654, NSF grant PHY-9513835 and the David and Lucile Packard Foundation.

\section{Appendix A. Flux stabilization at higher rank}

In this appendix we describe a generalization of the computation of [27] to higher rank groups. For some steps we specialize to $G=S U(N)$, but this restriction could probably easily be eliminated.

\section{A.1. Some volume computations}

In order to compare energies we will need volumes of groups and homogeneous spaces. We restrict attention to $G=S U(N)$.

If we identify $T_{e} G$ with the Lie algebra $\underline{\mathbf{g}}$ of traceless, $N \times N$ antihermitian matrices we will take the metric in this section to be

$$
g(X, Y)=-\operatorname{Tr}_{N}(X Y) \quad X, Y \in \underline{\mathbf{g}} .
$$

Put differently, we choose the metric

$$
d s^{2}=-\operatorname{Tr}_{N}\left[\left(g^{-1} d g\right) \otimes\left(g^{-1} d g\right)\right]
$$

$\underline{\text { Volume of } \mathrm{SU}(\mathrm{N})}$

We now compute the volume of $S U(N)$ in the metric (A.2). Consider $S U(N) / S U(N-$ $1)=S^{2 N-1}$. We relate the local coordinates of $S^{2 N-1}$ and $S U(N)$ via

$$
g=1+i y^{N} \operatorname{Diag}\{\epsilon, \ldots, \epsilon, 1\}+\sum_{i}\left(z^{i} e_{i N}-\bar{z}^{i} e_{N i}\right)+\cdots
$$


at $g=1$. (Here $\epsilon=-1 /(N-1)$. ). Here we are thinking of $S^{2 N-1}$ as the solutions to $\sum_{i=1}^{N}\left|z^{i}\right|^{2}=1$ and $z^{N}=x^{N}+i y^{N}$.

In these coordinates, the group metric (A.2) becomes

$$
d s^{2}=2 \sum_{i=1}^{N-1}\left[\left(d x^{i}\right)^{2}+\left(d y^{i}\right)^{2}\right]+\frac{N}{N-1}\left(d y^{N}\right)^{2}
$$

While (A.4) is not the round metric on the sphere it does give the invariant volume form on the sphere, up to an overall multiple. By left invariance we can compute this factor at the identity coset. Therefore, in the metric (A.2) we have

$$
\operatorname{vol}(S U(N) / S U(N-1))=\left(2^{N-1} \sqrt{\frac{N}{N-1}}\right) \operatorname{vol}\left(S^{2 N-1}\right)=\sqrt{\frac{N}{N-1}} \frac{(2 \pi)^{N}}{\Gamma(N)}
$$

(where we have embedded $S U(N-1) \hookrightarrow S U(N)$ with index 1).

Therefore

$$
\operatorname{vol}(S U(N))=\frac{\sqrt{N}}{2 \pi}(2 \pi)^{\frac{1}{2} N(N+1)} \frac{1}{1 ! 2 ! 3 ! \cdots(N-1) !}
$$

In particular,

$$
\operatorname{vol}(S U(3))=\frac{1}{2} \sqrt{3}(2 \pi)^{5}
$$

This checks against a formula in the literature [44, which was worked out in a slightly different way.

$\underline{\text { Volume of } \mathrm{G} / \mathrm{T}}$

The map $G / T \times T \rightarrow G$ is (generically) an $N$ !-fold covering. We compute the volume of $T \cong U(1)^{N-1}$ by taking standard orthonormal generators

$$
H_{j}=\sqrt{-1} \frac{1}{\sqrt{j(j+1)}} \operatorname{Diag}[1, \ldots, 1,-j, 0, \ldots, 0]
$$

so the range of angles is $0 \leq \theta_{j} \leq \sqrt{j(j+1)} 2 \pi$. Therefore

$$
\operatorname{vol}(T)=(N-1) ! \sqrt{N}(2 \pi)^{N-1}
$$

So $\operatorname{vol}(G / T)=N ! \operatorname{vol}(G) / \operatorname{vol}(T)$ gives

$$
\operatorname{vol}(G / T)=N(2 \pi)^{\frac{1}{2} N(N-1)} \frac{1}{1 ! 2 ! \cdots(N-1) !}
$$


One can rewrite this in a way that holds for all Lie groups. The positive roots are $e_{i}-e_{j}$ for $N \geq j>i \geq 1$. The fundamental weights are

$$
\lambda_{i}=e_{1}+\cdots+e_{i}-\frac{i}{N} \sum_{j=1}^{N} e_{j}
$$

The Weyl vector is:

$$
\rho=\sum_{j=1}^{N} \frac{1}{2}(N-(2 j-1)) e_{j}
$$

From this one computes

$$
\prod_{\alpha>0} \alpha \cdot \rho=1 ! 2 ! \cdots(N-1) !
$$

so we can write

$$
\operatorname{vol}(G / T)=N \prod_{\alpha>0} \frac{2 \pi}{\alpha \cdot \rho}
$$

One important subtlety is that the above is the metric on the space of left cosets $g T$. In our application we are interested in the adjoint group acting on the Lie algebra $g X g^{-1}$. Since the center of the group acts trivially we should compute the volume for $G=S U(N) / Z_{N}$. This gives

$$
\operatorname{vol}\left(G_{a d j} / T\right)=\prod_{\alpha>0} \frac{2 \pi}{\alpha \cdot \rho}
$$

\section{A.2. Flux stabilization at higher rank: Evaluation of the DBI action}

We will work with $S U(N)$. The rank $r=N-1$. The number of positive roots is $\Delta=\frac{1}{2} N(N-1)$.

We now choose coordinates

$$
g=k(\theta)^{-1} t(\chi) k(\theta)
$$

where $\chi$ are coordinates along the Cartan torus, and $\theta$ are angular coordinates along the space $G / T$. To be specific, we introduce a Cartan-Weyl basis

$$
\begin{aligned}
& {\left[H_{i}, H_{j}\right]=0} \\
& {\left[H_{i}, E_{\alpha}\right]=\alpha^{i} E_{\alpha}} \\
& {\left[E_{\alpha}, E_{\beta}\right]= \begin{cases}E_{\alpha+\beta} & \alpha+\beta \text { is a root } \\
0 & \text { otherwise }\end{cases} } \\
& {\left[E_{\alpha}, E_{\bar{\beta}}\right]= \begin{cases}\alpha^{i} H_{i} & \alpha=\beta \\
0 & \text { otherwise }\end{cases} }
\end{aligned}
$$


where the notation is that $\alpha, \beta, \ldots$ label positive roots and $\bar{\alpha}:=-\alpha$ etc. are the negative roots. We are also normalizing the generators so that

$$
\begin{aligned}
\operatorname{Tr}\left(H_{i} H_{j}\right) & =\delta_{i j} \\
\operatorname{Tr}\left(E_{\alpha} E_{\bar{\beta}}\right) & =\delta_{\alpha, \beta}
\end{aligned}
$$

Finally we set $t(\chi):=\exp \left[i \chi_{m} H_{m}\right]$ which are good coordinates transverse to the adjoint orbit $\mathcal{O}_{\chi}$ of $\exp [i \chi \cdot H]$ in $G$. Moreover we define

$$
d k k^{-1}=\theta^{\alpha} E_{\alpha}-\bar{\theta}^{\alpha} E_{\alpha}^{\dagger}+i \rho^{i} H_{i}
$$

The 1-forms $\theta^{\alpha}$ span a basis of holomorphic $(1,0)$ forms on $\mathcal{O}_{\chi}$ in the natural complex structure on $G / T$ induced by a choice of simple roots. Using

$$
g^{-1} d g=k^{-1} d k-(t k)^{-1} d k k^{-1}(t k)+k^{-1}\left(t^{-1} d t\right) k
$$

and a little algebra one can show that the 2 -form

$$
b=\frac{1}{4 \pi^{2} i} \sum_{\alpha>0}\left(\chi_{m} \alpha^{m}-\sin \left(\chi_{m} \alpha^{m}\right)\right) \theta^{\alpha} \wedge \bar{\theta}^{\alpha}
$$

precisely reproduces

$$
x_{3}=\frac{1}{24 \pi^{2}} \operatorname{Tr}_{N}\left(g^{-1} d g\right)^{3}=d b
$$

in an open neighborhood of the identity in a compact Lie group $G$.

To get normalizations straight, note that if $H=d B$ is an integral class and $F /(2 \pi)$ is an integral class then the worldsheet path integral looks like

$$
\exp \left[i \int_{\Sigma} 2 \pi B+F\right]
$$

So we should have :

$$
\frac{F}{2 \pi}+B=\frac{k}{2 \pi} \sum \sin (\chi \cdot \alpha) \frac{\theta_{\alpha} \wedge \theta_{\bar{\alpha}}}{2 \pi i}
$$

for $F=k \sum \chi \cdot \alpha \frac{\theta_{\alpha} \wedge \theta_{\bar{\alpha}}}{2 \pi i}$. Note that flux quantization restricts $\chi=2 \pi(\lambda+\rho) / k$, as expected.

In the parametrization (A.8) the orbit has metric induced by (A.2):

$$
-\left.\operatorname{Tr}_{N}\left(g^{-1} d g \otimes g^{-1} d g\right)\right|_{\mathcal{O}_{\chi}}=4 \sum_{\alpha>0} \sin ^{2}\left(\frac{1}{2} \alpha \cdot \chi\right)\left(\theta_{\alpha} \otimes \theta_{\bar{\alpha}}+\theta_{\bar{\alpha}} \otimes \theta_{\alpha}\right)
$$


As described below, the standard group metric (A.2) should be rescaled by $k / 2$ so

$g+2 \pi(F+2 \pi B)=2 k \sum_{\alpha>0}\left[\sin ^{2}\left(\frac{1}{2} \alpha \cdot \chi\right)\left(\theta_{\alpha} \otimes \theta_{\bar{\alpha}}+\theta_{\bar{\alpha}} \otimes \theta_{\alpha}\right)+i \frac{1}{2} \sin \alpha \cdot \chi\left(\theta_{\alpha} \otimes \theta_{\bar{\alpha}}-\theta_{\bar{\alpha}} \otimes \theta_{\alpha}\right)\right]$

and hence we get:

$$
d^{2 \Delta} \xi \sqrt{\operatorname{det}[g+2 \pi(F+2 \pi B)]}=(2 k)^{\Delta}\left(\prod_{\alpha>0} \sin \frac{1}{2} \alpha \cdot \chi\right) \operatorname{vol}\left(G_{a d j} / T\right)
$$

where $\operatorname{vol}\left(G_{a d j} / T\right)$ is the volume form for the adjoint orbit $G_{a d j} / T \subset \mathbf{g}$ in the metric (A.2).

Now, we have shown that wrt the metric (A.2) the volume of $G_{a d j} / T$ is

$$
\prod_{\alpha>0} \frac{2 \pi}{\alpha \cdot \rho}
$$

and so we get:

$$
\int_{\mathcal{O}_{\chi}} \sqrt{\operatorname{det}(g+F+B)}=(2 k)^{\Delta}\left(\prod_{\alpha>0} \sin \frac{1}{2} \alpha \cdot \chi\right) \prod_{\alpha>0} \frac{2 \pi}{\alpha \cdot \rho}
$$

\section{Remarks:}

1. Notice that the sin factors come in the first power in (A.17). This is to be contrasted with Weyl's formula for integrating class functions:

$$
\int_{G} f(g) d g=\int_{T} f(t) \prod_{\alpha>0} \sin ^{2}\left(\frac{1}{2} \alpha \cdot t\right) \frac{d t}{|W|}
$$

which implies that

$$
\sqrt{\operatorname{det} g} \sim\left(\prod_{\alpha>0} \sin ^{2} \frac{1}{2} \alpha \cdot \chi\right) \frac{1}{N !}
$$

The flux-stabilization mechanism effectively takes a squareroot of the volume!

2. The flux on this orbit is

$$
\frac{F}{2 \pi}=\sum_{\alpha>0}(\lambda+\rho) \cdot \alpha \frac{\theta_{\alpha} \wedge \theta_{\bar{\alpha}}}{2 \pi i}
$$

In the cohomology of $G / T$ this is $c_{1}\left(\mathcal{L}_{\lambda+\rho}\right)$ for the line bundle $\mathcal{L}_{\lambda+\rho} \rightarrow G / T$ one encounters in the Borel-Weil-Bott theorem. 


\section{A.3. Comparison with the CFT expression}

The normalization of the Minkowskian action for $S U(N)$ level $k$ WZW theory is

$$
S=\frac{k}{8 \pi} \int_{\Sigma} d x d y \operatorname{Tr}_{N}\left[\left(g^{-1} \partial_{x} g\right)^{2}+\left(g^{-1} \partial_{y} g\right)^{2}\right]+2 \pi k \int x_{3}
$$

Since the action is $\frac{1}{4 \pi \alpha^{\prime}} G_{\mu \nu} \partial X^{\mu} \partial X^{\nu}$ and we are taking $\alpha^{\prime}=1$ we have metric on the group $S U(N)$ given by

$$
g=-\frac{k}{2} \operatorname{Tr}_{N}\left(g^{-1} d g \otimes g^{-1} d g\right)
$$

for $S U(N)$.

Now we must multiply (A.19) by the tension of the D-brane, which is $1 /(2 \pi)^{2 \Delta}$, (in units with $\alpha^{\prime}=1$ ). To avoid confusions with string coupling and the normalization of the Newton constant, we actually will just compute the ratio of the mass of the brane to the mass of a D0 brane with $\lambda=0$. 14 The net result is

$$
\frac{\text { mass of D }- \text { brane on } \mathcal{O}_{\chi}}{\text { mass of D } 0-\text { brane }}=k^{\Delta}\left(\prod_{\alpha>0} \sin \frac{1}{2} \alpha \cdot \chi\right) \prod_{\alpha>0} \frac{1}{\pi \alpha \cdot \rho}
$$

Let us now compare this with the exact expression from conformal field theory. For $S U(N)$ we have the formula:

$$
S_{0, \lambda}=\frac{2^{\Delta}}{\sqrt{N}(k+N)^{r / 2}} \prod_{\alpha>0} \sin \left(\pi \frac{\alpha \cdot(\lambda+\rho)}{k+N}\right)
$$

Note that $S_{00} \sim k^{-\frac{1}{2} \operatorname{dim} G}$. Therefore, the mass of the Cardy state $\left.|\lambda\rangle\right\rangle_{C}$ is

$$
\frac{\left.\operatorname{Energy}(|\lambda\rangle\rangle_{C}\right)}{\left.\operatorname{Energy}(|0\rangle\rangle_{C}\right)}=\frac{S_{0 \lambda}}{S_{00}}=\prod_{\alpha>0} \frac{\sin \left(\pi \frac{\alpha \cdot(\lambda+\rho)}{k+N}\right)}{\sin \left(\pi \frac{\alpha \cdot \rho}{k+N}\right)}
$$

For $k \gg N$ we can expand the denominator in (A.27) to obtain

$$
(k+N)^{\Delta} \prod_{\alpha>0} \frac{1}{\pi \alpha \cdot \rho} \prod_{\alpha>0} \sin \left(\pi \frac{\alpha \cdot(\lambda+\rho)}{k+N}\right)
$$

Comparison with (A.25) shows that the leading $k$-dependence fits perfectly with the interpretation of a brane wrapping $G / T$ once.

14 We have also left out the boundary states for the fermions. These introduce extra factors of $\sqrt{2}$, but again cancel out of the ratio. 


\section{Appendix B. Volume of $M_{5}$}

\section{B.1. Integrally-normalized left-invariant forms}

All traces in this section are in the $N$ of $S U(N)$. We know that

$$
x_{3}=-\frac{1}{24 \pi^{2}} \operatorname{Tr}\left(g^{-1} d g\right)^{3}
$$

is the integral generator of $H^{3}(S U(N) ; Z)$. In general the spherical class in $\pi_{2 k+1}(S U(N))$ is $k$ ! times the primitive generator of $H_{2 k+1}(S U(N))$ for $N$ in the stable range [45, 46, 47]. Using this and the ABS construction one can find the integral normalization of the higher traces. In particular we have the integral class

$$
x_{5}=-2 \frac{2 !}{(2 \pi i)^{3} 5 !} \operatorname{Tr}\left(g^{-1} d g\right)^{5}
$$

In the coordinates

$$
g=\exp \left[i \theta_{1} H_{1}+i \theta_{2} H_{2}+\sum_{\alpha>0}\left(z_{\alpha} E_{\alpha}-\bar{z}_{\alpha} E_{\bar{\alpha}}\right)\right]
$$

we find near the identity

$$
\begin{aligned}
\operatorname{Tr}\left(g^{-1} d g\right)^{5} & =\frac{15 i}{\sqrt{2}} d \theta_{1}\left(d^{2} z_{1} d^{2} z_{2}+d^{2} z_{1} d^{2} z_{3}\right) \\
& +\frac{15 i}{\sqrt{6}} d \theta_{2}\left(d^{2} z_{1} d^{2} z_{3}-2 d^{2} z_{2} d^{2} z_{3}-d^{2} z_{1} d^{2} z_{2}\right) \\
& -\frac{15}{\sqrt{3}} d \theta_{1} d \theta_{2}\left(d z_{1} d z_{2} d \bar{z}_{3}-d \bar{z}_{1} d \bar{z}_{2} d z_{3}\right) \\
& +\operatorname{Tr} p^{5}
\end{aligned}
$$

where $d^{2} z:=d z \wedge d \bar{z}$. Here $p=d z_{\alpha} E_{\alpha}-d \bar{z}_{\alpha} E_{\bar{\alpha}}$.

Now, since $x_{5}$ restricts to the left-invariant unit volume form on $M_{5}$ we can compare with that induced by the metric (A.2). From (B.4) we get

$$
\left.x_{5}\right|_{M_{5}}=-\frac{4}{(2 \pi)^{3} 5 !} \frac{30}{\sqrt{3}} d \theta_{1} d \theta_{2} d y_{1} d y_{2} d y_{3}
$$

whereas the Haar measure in the metric (A.2) is $\sqrt{8} d \theta_{1} d \theta_{2} d y_{1} d y_{2} d y_{3}$. From this we deduce that the induced volume on $M_{5} \subset S U(3)$ is

$$
\operatorname{vol}\left(M_{5}\right)=\frac{4}{\sqrt{2}} \sqrt{3}(2 \pi)^{3}
$$




\section{Appendix C. Proof of an arithmetic identity}

We would like to prove that

$$
\frac{k}{\operatorname{gcd}(k, \operatorname{lcm}(1,2, \ldots, N))}
$$

is the same as

$$
\operatorname{gcd}\left[\left(\begin{array}{l}
k \\
1
\end{array}\right),\left(\begin{array}{l}
k \\
2
\end{array}\right), \ldots,\left(\begin{array}{c}
k \\
N
\end{array}\right)\right]
$$

Consider the prime divisors of $k$ and of $1,2, \ldots, N$. For each prime $p$ and integer $n$ write $n=p^{v_{p}(n)} n^{\prime}$ where $n^{\prime}$ is relatively prime to $p$. We will compare the prime powers in (C.1) and (C.2) and show that they are the same.

We consider 3 cases:

1. If $v_{p}(k)=0$ then both factors obviously have $p^{0}$.

2. If $v_{p}(k)>0$ and $v_{p}(k)>v_{p}(j)$ for all $j=1,2, \ldots, N$ then note that

$$
\left(\begin{array}{l}
k \\
j
\end{array}\right)=\frac{k}{j} \frac{k-1}{1} \frac{k-2}{2} \cdots \frac{k-(j-1)}{j-1}= \pm \frac{k}{j}(1-k)(1-k / 2) \cdots\left(1-\frac{k}{j-1}\right)
$$

The first factor has $p^{v_{p}(k)-v_{p}(j)}$ while the others are of the form $1+p^{\text {positive }}$, and the coefficients of $p^{\text {positive }}$ are fractions whose denominators are prime to $p$. Therefore $v_{p}\left(\left(\begin{array}{c}k \\ j\end{array}\right)\right)=$ $v_{p}(k)-v_{p}(j)$.

Now

$$
\begin{aligned}
\min _{j=1,2, \ldots, N}\left(v_{p}(k)-v_{p}(j)\right) & =v_{p}(k)-\max _{j=1, \ldots, N}\left(v_{p}(j)\right) \\
& =v_{p}(k)-\min \left[v_{p}(k), \max _{j=1, \ldots, N}\left(v_{p}(j)\right)\right]
\end{aligned}
$$

establishes the identity.

3. Finally, suppose that for some $j \leq N, v_{p}(j) \geq v_{p}(k)>0$. Clearly, there is a $j^{\prime} \leq j$ with $v_{p}\left(j^{\prime}\right)=v_{p}(k)$. We claim that for this $j^{\prime}$ we have $v_{p}\left(\left(\begin{array}{l}k \\ j^{\prime}\end{array}\right)\right)=0$. This again follows from (C.3) together with the observation that every $j^{\prime \prime}<j^{\prime}$ has $v_{p}\left(j^{\prime \prime}\right)<v_{p}(k)$. Therefore, for such primes the power of $p$ in (C.1) and (C.2) is $p^{0}$.

Thus (C.1) is equal to (C.2) 


\section{References}

[1] R. Minasian and G. Moore, "K-Theory and Ramond-Ramond Charge," JHEP 9711: 002, 1997; hep-th/9710230.

[2] E. Witten, " $D$-Branes And $K$-Theory," JHEP 9812:019, 1998; hep-th/9810188.

[3] A. Sen, "Tachyon condensation on the brane antibrane system," JHEP 9808, 012 (1998) hep-th/9805170.

[4] D.S. Freed and E. Witten, "Anomalies in String Theory with $D$-branes," hepth/9907189.

[5] D. Freed, "Dirac Charge Quantization and Generalized Differential Cohomology," hepth/0011220

[6] E. Diaconescu, G. Moore, and E. Witten, " $E_{8}$ Gauge Theory, and a Derivation of $K$-Theory from $M$-Theory," hep-th/0005090

[7] E. Witten "Baryons And Branes In Anti de Sitter Space," hep-th/9805112; JHEP 9807 (1998) 006

[8] A. Kapustin, " $D$-branes in a Topologically Nontrivial B-field," hep-th/9909089.

[9] P. Bouwknegt and V. Mathai, " $D$-Branes, $B$ Fields and Twisted $K$-Theory," JHEP 0003:007,2000, hep-th/0002023.

[10] V. Mathai and I.M. Singer, "Twisted K-homology theory, twisted Ext-theory," hepth/0012046

[11] P. Bouwknegt, A. L. Carey, V. Mathai, M. K. Murray, D. Stevenson, "Twisted Ktheory and K-theory of bundle gerbes," hep-th/0106194

[12] E. Witten, "Overview of K-theory applied to strings," hep-th/0007175.

[13] J. Harvey and G. Moore, "Noncommutative Tachyons and K-Theory," hep-th/0009030.

[14] J. Maldacena, G. Moore, and N. Seiberg, "D-brane charges in five-brane backgrounds," to appear

[15] J. Maldacena, G. Moore, and N. Seiberg, "Geometrical interpretation of D-branes in gauged WZW models," hep-th/0105038

[16] H. Cartan and S. Eilenberg, Homological Algebra, ch. XV

[17] M.F. Atiyah and F. Hirzebruch, "Vector Bundles and Homogeneous Spaces," Proc. Symp. Pure Math. 3 (1961) 53.

[18] J. Rosenberg, "Homological Invariants of Extensions of $C^{*}$-algebras," Proc. Symp. Pure Math 38 (1982) 35.

[19] M. Atiyah and G.B. Segal, unpublished.

[20] O. Bergman, E. Gimon, and S. Sugimoto, "Orientifolds, RR torsion, and K-theory," hep-th/0103183

[21] I. Affleck and A. Ludwig, "The Kondo Effect, Conformal Field Theory, and Fusion Rules," Nucl.Phys.B352:849-862,1991 ; "Critical Theory of Overscreened Kondo Fixed Points," Nucl.Phys.B360:641-696,1991 
[22] A. Alekseev and V. Schomerus, "D-branes in the WZW model," hep-th/9812193; Phys. Rev. D60(1999)061901

[23] K. Gawedzki, "Conformal field theory: a case study," hep-th/9904145

[24] S. Stanciu, "D-branes in group manifolds," hep-th/9909163

[25] G. Felder, J. Fröhlich, J. Fuchs, C. Schweigert, "The geometry of WZW branes,"hepth/9909030, J.Geom.Phys. 34 (2000) 162-190

[26] A. Alekseev, A. Recknagel, and V. Schomerus, "Non-commutative World-volume Geometries: Branes on SU(2) and Fuzzy Spheres," hep-th/9908040; "Brane Dynamics in Background Fluxes and Non-commutative Geometry," hep-th/0003187

[27] C. Bachas, M. Douglas, and C. Schweigert, "Flux Stabilization of D-branes,"hepth/0003037

[28] J. Fuchs and C. Schweigert, "A classifying algebra for boundary conditions," hepth/9708141, Phys.Lett. B414 (1997) 251-259 ;

"Branes: from free fields to general backgrounds," Nucl. Phys. B530 (1998) 99, hepth/9712257;

"Symmetry breaking boundaries I. General theory," hep-th/9902132;

"Symmetry breaking boundaries. II: More structures, examples," Nucl. Phys. B 568 (2000) 543 hep-th/9908025.

[29] A. Alekseev and V. Schomerus, "RR charges of D2-branes in the WZW model," hepth/0007096

[30] L. Birke, J. Fuchs, and C. Schweigert, "Symmetry breaking boundary conditions and WZW orbifolds," Adv. Theor. Math. Phys. 3, 671 (1999); hep-th/9905038

[31] J. Fuchs, B. Schellekens, and C. Schweigert, "From Dynkin diagram symmetries to fixed point structures," hep-th/9506135

[32] M. R. Douglas, D. Kabat, P. Pouliot and S. H. Shenker, "D-branes and short distances in string theory," Nucl. Phys. B 485, 85 (1997) hep-th/9608024.

[33] S. Fredenhagen and V. Schomerus, "Branes on Group Manifolds, Gluon Condensates, and twisted K-theory," hep-th/0012164

[34] S. Elitzur, G. Moore, A. Schwimmer and N. Seiberg, "Remarks On The Canonical Quantization Of The Chern-Simons-Witten Theory," Nucl. Phys. B 326, 108 (1989).

[35] R. C. Myers, "Dielectric-branes," JHEP 9912, 022 (1999) hep-th/9910053.

[36] D. Friedan, Z. Qiu, and S.H. Shenker, Phys. Lett. 151B(1985)37

[37] P. di Vecchia, V.G. Knizhnik, J.L. Petersen, and P. Rossi, Nucl. Phys. B253(1985) 701

[38] V.G. Kac and I.T. Todorov, Comm. Math. Phys. 102(1985)337

[39] P, Di Francesco, P. Mathieu, D. Sénégal, Conformal Field Theory Springer, 1997

[40] J. Fuchs, Affine Lie Algebras and Quantum Groups, Cambridge Univ. Press

[41] R. Wendt, "Weyl's character formula for non-connected Lie groups and orbital theory for twisted affine Lie algebras," math.RT/9909059 
[42] M. Hopkins, Unpublished result. July, 2001

[43] Epstein and N. Steenrod, Cohomology Operations, Ann. Math. Stud. 50, Princeton Univ. Press

[44] K. Abe and I. Yokota, "Volumes of compact symmetric spaces," Tokyo J. Math. 20 (1997) 87

[45] R. Bott, "A note on the Samelson product in the classical groups," Comment. Math. Helv. 34 (1960). See Collected Works, vol. 1

[46] R. Bott, "The space of loops on a Lie group," Mich. Jour. Math. 5(1958) 35

[47] L. Smith, Topology 8 (1969)69; Kane and Moreno, Can. J. Math. 6 (1988) 1331 